\title{
Titanato de circonio: estabilidad termodinámica y expansión térmica
}

\author{
E. LÓPEZ-LÓPEZ, R. MORENO, C. BAUDÍN* \\ Instituto de Cerámica y Vidrio, CSIC. Kelsen 5, 28049, Madrid, España. * cbaudin@icv.csic.es
}

\begin{abstract}
El titanato de circonio es un compuesto muy usado en aplicaciones electrocerámicas, aunque también se han descrito aplicaciones en el campo de la catálisis y de los sensores. Dada la anisotropía en la expansión térmica cristalográfica de este compuesto, podría ser planteado como constituyente de componentes estructurales. En general, para asegurar la integridad estructural y la homogeneidad microestructural de una pieza cerámica, es preciso utilizar velocidades de enfriamiento desde la temperatura de fabricación relativamente bajas. Este requerimiento tiene una importancia fundamental en el titanato de circonio, ya que pequeñas variaciones en la composición y en la velocidad de enfriamiento, producen variaciones significativas tanto en la distribución de fases como en la expansión térmica. En este trabajo se revisan los trabajos existentes sobre la estabilidad del titanato de circonio dentro de los sistemas $\mathrm{ZrO}_{2}-\mathrm{TiO}_{2}$ y $\mathrm{ZrO}_{2}-\mathrm{TiO}_{2}-\mathrm{Y}_{2} \mathrm{O}_{3}$. Se describen las principales discrepancias acerca de las fases compatibles existentes en la bibliografía actual y se discute el posible origen de estas discrepancias. Asimismo, se revisan los datos existentes sobre la expansión térmica cristalográfica de este compuesto.
\end{abstract}

Palabras clave: Diagramas de fases, Reactividad, Propiedades térmicas, Aplicaciones estructurales, Titanato de circonio, sistema $\mathrm{ZrO}_{2}-$ $\mathrm{TiO}_{2}-\mathrm{Y}_{2} \mathrm{O}_{3}$

\section{Zirconium titanate: stability and thermal expansion}

Zirconium titanate is a well known compound in the field of electroceramics, although it has also been used in catalyst and sensors applications. The crystallographic thermal expansion anisotropy of this compound makes it a potential candidate as constituent of structural components. In general, to assure the structural integrity and microstructural homogeneity of a ceramic piece, relatively low cooling rates from the fabrication temperature are required. This requirement is essential for zirconium titanate because thermal expansion as well as phase distribution is affected by small variations in the composition and cooling rate. This work reviews the available data on the phase equilibrium relationships in the systems $\mathrm{ZrO}_{2}-\mathrm{TiO}_{2}$ and $\mathrm{ZrO}-\mathrm{TiO}_{2}-\mathrm{Y}_{2} \mathrm{O}_{3}$. The main discrepancies as well as the possible origins of them are discussed. Additionally, the crystallographic thermal expansion data in the current literature are reviewed.

Key words: Phase diagrams, Reactivity, Thermal properties, Structural applications, Zirconium titanate, $\mathrm{ZrO}_{2}-\mathrm{TiO}_{2}-\mathrm{Y}_{2} \mathrm{O}_{3}$ system

\section{INTRODUCCIÓN}

El titanato de circonio $\left(\mathrm{Zr}_{\mathrm{x}} \mathrm{Ti}_{1-\mathrm{x}}\right)_{2} \mathrm{O}_{4^{\prime}}$ compuesto intermedio dentro del sistema $\mathrm{ZrO}_{2}-\mathrm{TiO}_{2}[1,2]$ forma una solución sólida que abarca composiciones en las que la fracción molar de Ti, (1-x), varía entre 0.42-0.67, dependiendo de las condiciones de presión y temperatura [3]. El mineral srilankita $\left(\mathrm{ZrTi}_{2} \mathrm{O}_{6}\right)[4,5]$ y los compuestos sintéticos $\mathrm{ZrTiO}_{4}$ [6] y $\mathrm{Zr}_{5} \mathrm{Ti}_{7} \mathrm{O}_{24}[4,7]$ son composiciones específicas dentro de esta solución sólida. En los diferentes estudios realizados a presión atmosférica que se describen en este trabajo, se mencionan las composiciones estequiométricas $\mathrm{ZrTiO}_{4^{\prime}} \mathrm{ZrTi}_{2} \mathrm{O}_{6}$ y $\mathrm{Zr}_{5} \mathrm{Ti}_{7} \mathrm{O}_{24^{\prime}}$ así como soluciones sólidas de $\mathrm{TiO}_{2}$ y de $\mathrm{ZrO}_{2}$ en $\mathrm{ZrTiO}_{4}$ y $\mathrm{ZrTi}_{2} \mathrm{O}_{6}$. Por ello, si bien la fórmula química $\left(\mathrm{Zr}_{\mathrm{x}} \mathrm{Ti}_{1-\mathrm{x}}\right)_{2} \mathrm{O}_{4}$ describe correctamente todas las posibles composiciones, en este trabajo, por claridad, se utilizará la nomenclatura tradicional empleada para la descripción de las soluciones sólidas: $\mathrm{ZrTiO}_{4}$ ss y $\mathrm{ZrTi}_{2} \mathrm{O}_{6} \mathrm{ss}$.
El compuesto $\mathrm{ZrTiO}_{4}{ }^{1}$ cristaliza en el sistema ortorrómbico con una estructura tipo $\alpha-\mathrm{PbO}_{2}$. Los átomos de circonio y titanio se distribuyen al azar ocupando sitios octaédricos $[6,8,9]$. El ámbito tradicional de los materiales de titanato de circonio es el de la electrocerámica, como resonadores dieléctricos debido a su elevada constante dieléctrica $(\kappa \approx 35-46)$ [10-14], a su alto factor de calidad $(Q \approx 2000-8000)$ medido entre 1 y $10 \mathrm{GHz}[10-14]$ y a su pequeña variación de la frecuencia de resonancia con la temperatura $\left(\tau_{\mathrm{f}} \approx 0 \mathrm{ppm}^{\circ} \mathrm{C}^{-1}\right)[10,11,14]$. Estas propiedades hacen que los materiales de titanato de circonio sean usados en el campo de las telecomunicaciones como sintonizadores, filtros y osciladores de tensión sintonizable mediante voltaje $[10,15]$. Además, se han determinado las propiedades dieléctricas del titanato de circonio en películas delgadas preparadas por ablación láser [15].

1 Estructura cristalina: ortorrómbica.Grupo espacial: Pnab. $\mathrm{a}=5.03580 \mathrm{~nm}, \mathrm{~b}=$ $5.48740 \mathrm{~nm}, \mathrm{c}=4.80180 \mathrm{~nm}$. (PDF: 00-034-0415) 
Por otro lado, las mezclas binarias de $\mathrm{ZrO}_{2}-\mathrm{TiO}_{2}$, tanto sin reaccionar como formando titanato de circonio, se utilizan en aplicaciones catalíticas [16-20]. El titanato de circonio tiene actividad catalítica en algunas reacciones como la deshidrogenación no oxidativa del etilbenceno [17, 20], el cual tiene importancia en la producción de estireno. Así mismo, se ha utilizado en un gran número de reacciones catalíticas como soporte de catalizadores [17]. Además, el titanato de circonio tiene actividad fotocatalítica [16, 17]. También se han descrito aplicaciones del titanato de circonio como pigmento [21] y como sensor de hidrocarburos (metano y propano) $[17,22]$ y de humedad [23].

De acuerdo con los cálculos realizados por Hom y col. [24], la formación de $\mathrm{ZrTiO}_{4}$ a partir de $\mathrm{ZrO}_{2}$ y $\mathrm{TiO}_{2}$ es termodinámicamente favorable a temperaturas superiores a $980^{\circ} \mathrm{C}\left(1250 \pm 150^{\circ} \mathrm{K}\right)$. De hecho, distintos autores han reportado temperaturas de síntesis de $\mathrm{ZrTiO}_{4}$ por reacción en estado sólido de polvos de $\mathrm{ZrO}_{2} \mathrm{y} \mathrm{TiO}_{2}$ entre $\approx 1000-1700^{\circ} \mathrm{C}[4$, 6, 8-10, 14, 24-27]. Los métodos sol-gel [28], permiten obtener $\mathrm{ZrTiO}_{4}$ por tratamiento térmico a temperaturas de $500-600^{\circ} \mathrm{C}$. La mayoría de los trabajos describen métodos de fabricación de polvos o piezas pequeñas, no habiéndose descrito la fabricación de piezas masivas $\left(\approx 60 \times 60 \times 5 \mathrm{~mm}^{3}\right)$ hasta fecha reciente [29-31].

\section{SISTEMA $\mathrm{ZrO}_{2}-\mathrm{TiO}_{2}$}

Los primeros estudios del sistema $\mathrm{ZrO}_{2}-\mathrm{TiO}_{2}$ fueron realizados por Sowman y Andrews en 1951 [32]. Estos autores no detectaron la presencia de $\mathrm{ZrTiO}_{4}$ y establecieron que a $\mathrm{T}$ $\approx 1600^{\circ} \mathrm{C}$ el límite de solubilidad en estado sólido de $\mathrm{ZrO}_{2}$ en $\mathrm{TiO}_{2}$ era $\approx 21 \%$ peso $(\approx 14.7 \% \mathrm{~mol})$, y el de $\mathrm{TiO}_{2}$ en $\mathrm{ZrO}_{2}$ $\approx 37 \%$ peso $(\approx 47.4 \% \mathrm{~mol})$, concluyendo que el sistema $\mathrm{ZrO}_{2}-$ $\mathrm{TiO}_{2}$ estaba formado por soluciones sólidas parciales sin compuestos intermedios. Además, propusieron un punto eutéctico localizado entre $50-55 \%$ peso $(39.3-44.2 \% \mathrm{~mol})$ de $\mathrm{ZrO}_{2}$ a $1600^{\circ} \mathrm{C}$.

En 1954, de manera independiente Brown y Duwez [1] y Coughanour y col. [2], publicaron por primera vez la existencia de un nuevo compuesto de fórmula $\mathrm{ZrTiO}_{4}$. Además, Coughanour y col. [2] establecieron que este compuesto cristalizaba en el sistema ortorrómbico y fueron los primeros en proponer la existencia de dos polimorfos de este compuesto con una temperatura de transición comprendida entre $\approx 800-1200^{\circ} \mathrm{C}$ para explicar las diferencias existentes en la longitud del eje cristalográfico b (eje c según la convención usada por Coughanour y col. [2]) en función de la velocidad de enfriamiento.

Brown y Duwez [1] estudiaron mezclas binarias de $\mathrm{TiO}_{2}$ y $\mathrm{ZrO}_{2}$ tratadas a distintas temperaturas y propusieron un diagrama de equilibrio de fases (Fig. 1) en el cual la máxima solubilidad de $\mathrm{ZrO}_{2}$ en $\mathrm{TiO}_{2}$ a $\mathrm{T} \approx 1760^{\circ} \mathrm{C}$ era aproximadamente $18 \%$ mol y decrecía hasta aproximadamente $6 \%$ mol a $\mathrm{T} \approx$ $980^{\circ} \mathrm{C}$. Además, pusieron de manifiesto cómo a medida que aumentaba la cantidad de $\mathrm{TiO}_{2}$ en solución sólida en $\mathrm{ZrO}_{2}$, la temperatura de transformación de la fase tetragonal $\left(\mathrm{t}-\mathrm{ZrO}_{2}\right)$ a la monoclínica $\left(\mathrm{m}-\mathrm{ZrO}_{2}\right)$ se reducía considerablemente hasta llegar a $\mathrm{T} \approx 340^{\circ} \mathrm{C}$ para un máximo de solubilidad de aproximadamente $40 \%$ mol de $\mathrm{TiO}_{2}$ en $\mathrm{t}-\mathrm{ZrO}_{2}$.

Las relaciones de equilibrio de fases entre $1600^{\circ} \mathrm{C}$ y $1900^{\circ} \mathrm{C}$ (Fig. 2) propuestas por Coughanour y col. [2] son

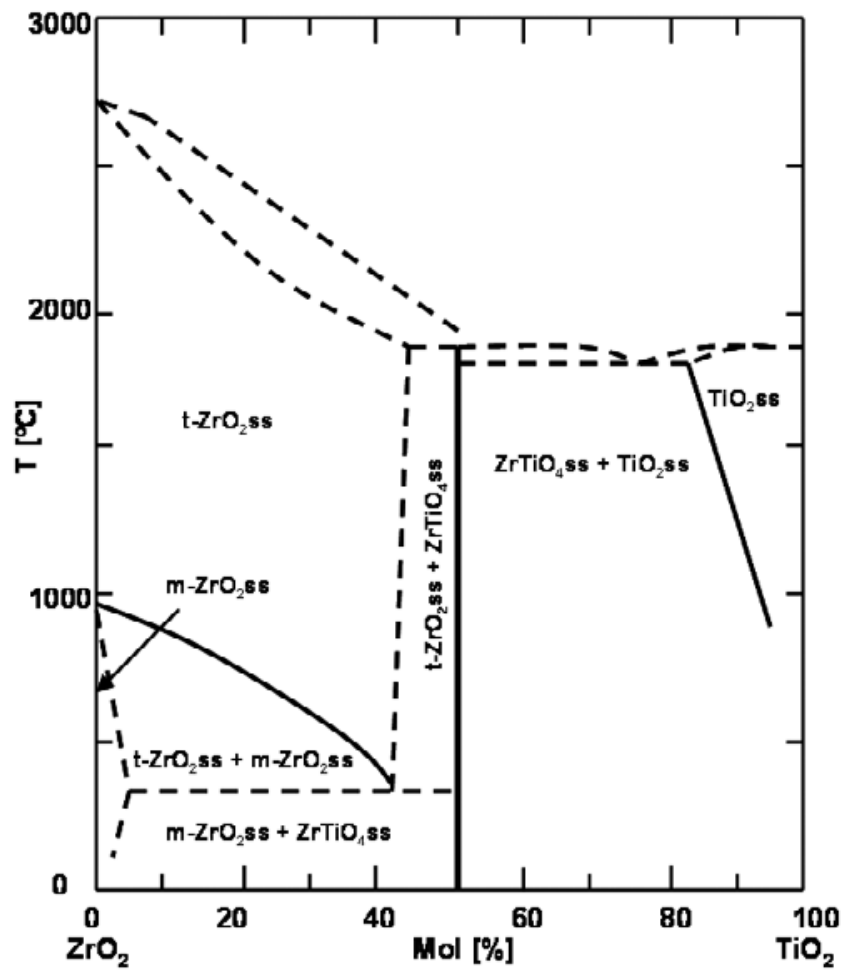

Figura 1. Diagrama de equilibrio de fases del sistema $\mathrm{ZrO}_{2}-\mathrm{TiO}_{2}$ publicado por Brown y Duwez en 1954 [1].

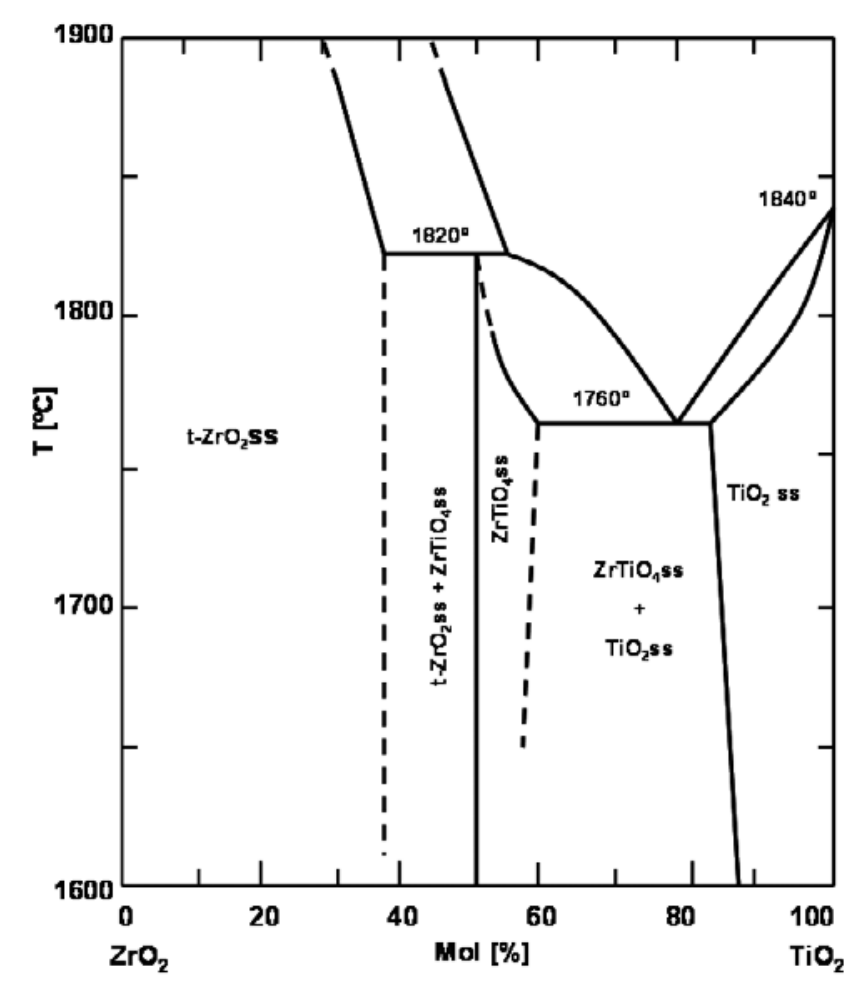

Figura 2. Diagrama de equilibrio de fases del sistema $\mathrm{ZrO}_{2}-\mathrm{TiO}_{2}$ publicado por Coughanour y col. en 1954 [2]. 
muy similares a las que propusieron Brown y Duwez [1] (Fig. 1). Coughanour y col. [2] fijaron las líneas de solidus y de liquidus a partir del establecimiento de los puntos de fusión de los compuestos puros y de las temperaturas de solidus y de liquidus de mezclas binarias de $\mathrm{ZrO}_{2} \mathrm{y} \mathrm{TiO}_{2}$. Estas líneas sólo habían sido dibujadas en tentativa por Brown y Duwez [1].

En 1968, Noguchi y Mizuno [33] realizaron un estudio detallado de las líneas de liquidus para composiciones comprendidas entre $2.5 \%$ y $20 \%$ mol de $\mathrm{TiO}_{2}$. Este estudio, al igual que Coughanour y col. [2], lo realizaron a partir del establecimiento de los puntos de fusión de los compuestos puros y de las temperaturas de solidus y de liquidus de las mezclas binarias. A partir del límite de solubilidad sólida de $\mathrm{TiO}_{2}$ en $\mathrm{t}-\mathrm{ZrO}_{2}$ determinado a $1700^{\circ} \mathrm{C}\left(17.5 \%\right.$ mol de $\mathrm{TiO}_{2}$ en $\mathrm{t}-\mathrm{ZrO}_{2}$ ) y de trabajos anteriores de otros autores [34] propusieron un diagrama de fases en tentativa (Fig. 3) en el que los límites de solubilidad sólida de $\mathrm{TiO}_{2}$ en $\mathrm{t}-\mathrm{ZrO}{ }_{2}$ son muy inferiores a los propuestos en los trabajos anteriormente mencionados $[1,2,32]$ y establecieron una región monofásica de $\mathrm{ZrTiO}_{4} \mathrm{ss}$ muy estrecha en la zona rica en $\mathrm{TiO}_{2}$ (50-53\% mol $\mathrm{TiO}_{2}$ ). Para satisfacer la regla de las fases, en el diagrama propuesto se exigía la existencia de un nuevo polimorfo de $\mathrm{ZrO}_{2}$ a temperaturas superiores a $2500^{\circ} \mathrm{C}$.

En 1980 Shevchenko y col. [35] estudiaron el diagrama $\mathrm{ZrO}_{2}-\mathrm{TiO}_{2}$ a temperaturas superiores a $1600^{\circ} \mathrm{C}$ (Fig. 4). $\mathrm{El}$ análisis de muestras cristalinas de composiciones con contenidos crecientes de $\mathrm{TiO}_{2}(0-100 \%$ mol $)$ a $1700^{\circ} \mathrm{C}$ les permitió ajustar la región monofásica de $\mathrm{ZrTiO}_{4} \mathrm{ss}$ a $1700^{\circ} \mathrm{C}$, propuesta en tentativa por otros autores [1, 2, 33], entre 40.0-

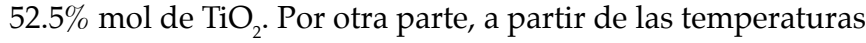
de fusión de los compuestos puros y de las temperaturas

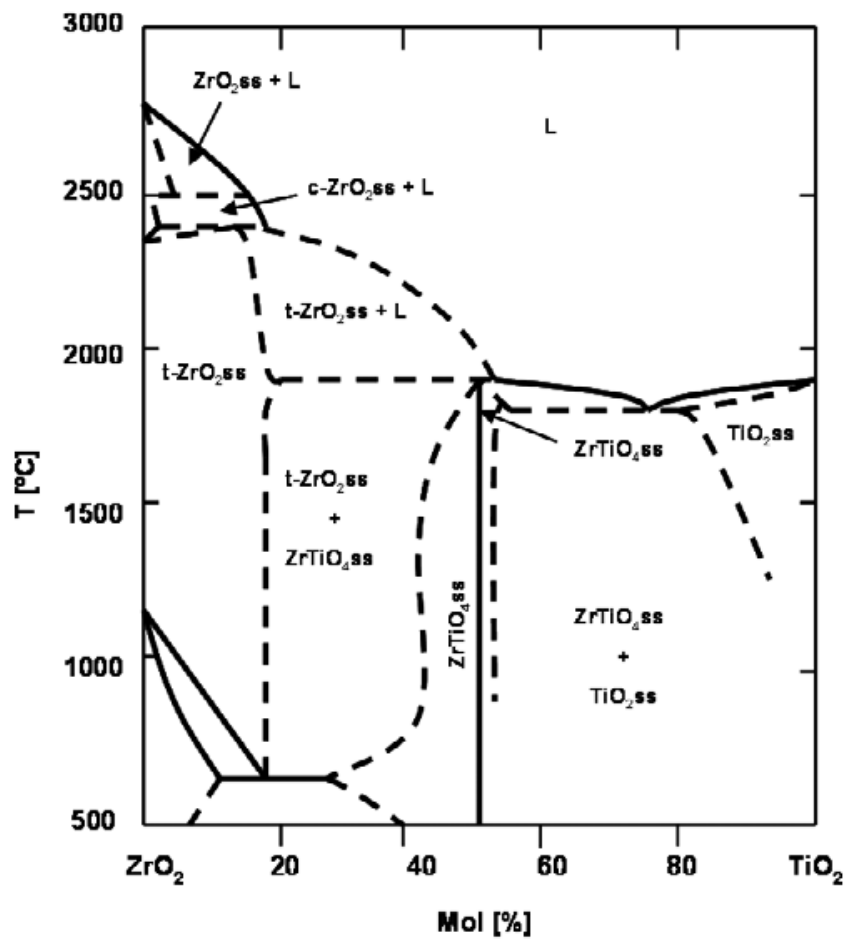

Figura 3. Diagrama de equilibrio de fases del sistema $\mathrm{ZrO}_{2}-\mathrm{TiO}_{2}$ publicado por Noguchi y Mizuno en 1968 [33] de solidus y de liquidus de mezclas binarias de $\mathrm{TiO}_{2}$ y $\mathrm{ZrO}_{2}$ establecieron la línea de líquidus con tres puntos invariantes, dos peritécticos $\left(\approx 2200^{\circ} \mathrm{C}\right.$ y $\left.1830 \pm 20^{\circ} \mathrm{C}\right)$ y un eutéctico $(1720$ $\left.\pm 20^{\circ} \mathrm{C}\right)$. De acuerdo con estos autores la fusión de $\mathrm{ZrTiO}_{4}$ es incongruente $\left(1830 \pm 20^{\circ} \mathrm{C}\right)$.

En 1986, Bannister y Barnes [36] estudiaron la zona rica en $\mathrm{ZrO}_{2}$ (hasta $30 \%$ mol de $\mathrm{TiO}_{2}$ ) del sistema $\mathrm{ZrO}_{2}-\mathrm{TiO}_{2}$, y determinaron que la solubilidad máxima de $\mathrm{TiO}_{2}$ en $\mathrm{t}-\mathrm{ZrO}_{2}$ a $1300^{\circ} \mathrm{C}$ era $13.8 \pm 0.3 \% \mathrm{~mol}$, a $1400^{\circ} \mathrm{C} 14.9 \pm 0.2 \%$ mol y a $1500^{\circ} \mathrm{C}$ $16.1 \pm 0.2 \%$ mol. Al igual que Brown y Duwez [1], Bannister y Barnes [36] pusieron de manifiesto que la solución sólida de $\mathrm{TiO}_{2}$ en $\mathrm{ZrO}_{2}$ disminuye la temperaturas de transformación de $\mathrm{t}-\mathrm{ZrO}_{2}$ a $\mathrm{m}-\mathrm{ZrO}_{2}$. En particular, determinaron la temperatura de transformación de $\mathrm{t}-\mathrm{ZrO}_{2}$ a $\mathrm{m}-\mathrm{ZrO}_{2^{\prime}} \approx 775 \mathrm{y} \approx 675^{\circ} \mathrm{C}$ (valores calculados a partir de la representación gráfica incluida en la referencia [36]), para composiciones de $\mathrm{ZrO}_{2} \mathrm{ss}$ con 13.8 y $16.1 \%$ mol de $\mathrm{TiO}_{2}$, respectivamente.

Entre 1983 y 1986, McHale y Roth [4, 37] estudiaron composiciones binarias de $\mathrm{ZrO}_{2}$ y TiO $\mathrm{Ti}_{2}$ con proporciones de $\mathrm{ZrO}_{2}$ que oscilaban entre 40 y $60 \%$ mol tratadas a $1500^{\circ} \mathrm{C}$ y con tratamientos isotermos a temperaturas comprendidas entre 1000 y $1200^{\circ} \mathrm{C}$ durante el enfriamiento. Estos autores observaron que, en muestras de $\mathrm{ZrTiO}_{4}$ tratadas durante 4 meses a $\mathrm{T} \approx 1000^{\circ} \mathrm{C}$, las fases presentes a temperatura ambiente eran una mezcla de $\mathrm{m}-\mathrm{ZrO}_{2} \mathrm{ss}$ y una nueva fase donde la relación molar $\mathrm{Zr} / \mathrm{Ti}$ era $1 / 2$, siendo su fórmula $\mathrm{ZrTi}_{2} \mathrm{O}_{6}$. Por lo tanto, esta nueva fase sería la fase estable a baja temperatura en el sistema $\mathrm{ZrO}_{2}-\mathrm{TiO}_{2}$ en ausencia de impurezas. Coincidiendo con los estudios de McHale y Roth $[4,37]$ se descubrió un nuevo mineral denominado srilankita con la misma composición $\left(\mathrm{ZrTi}_{2} \mathrm{O}_{6}\right)[5,38]$, lo cual apoyó las

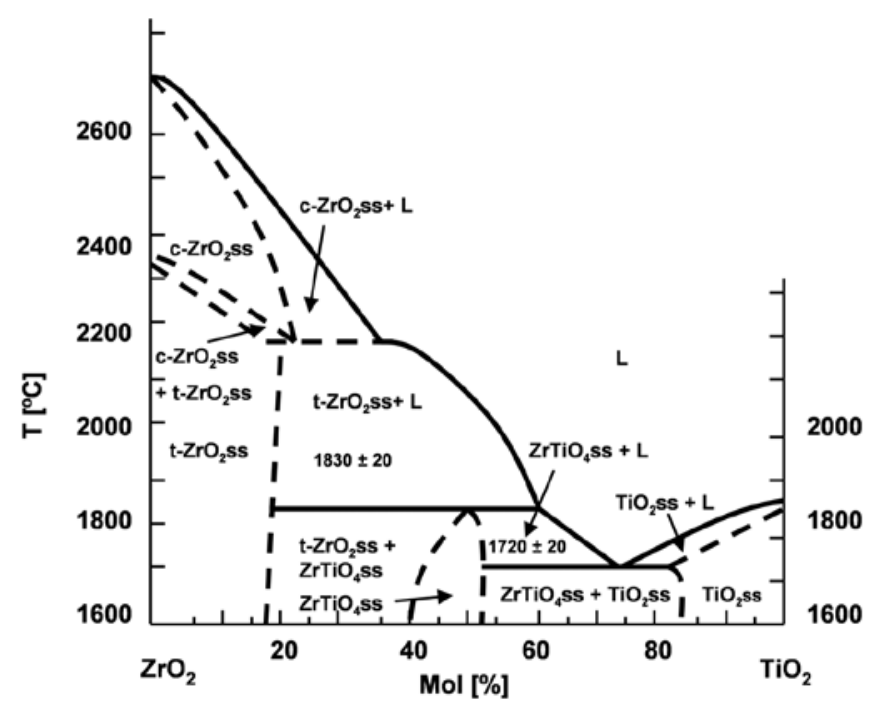

Figura 4. Diagrama de equilibrio de fases del sistema $\mathrm{ZrO}_{2}-\mathrm{TiO}_{2}$ publicado por Shevchenko y col. [35]. 
conclusiones de estos autores [4]. La estructura cristalina de $\mathrm{ZrTi}_{2} \mathrm{O}_{6}$ es ortorrómbica, al igual que la de $\mathrm{ZrTiO}_{4^{\prime}}$ y presenta una superestructura en el eje c (eje a de de acuerdo con la convención usada por McHale y Roth [4]).

De acuerdo con el diagrama de fases propuesto por McHale y Roth [4] (Fig. 5) a $\mathrm{T} \approx 1100^{\circ} \mathrm{C}$ hay un punto eutectoide que indica la descomposición de $\mathrm{ZrTiO}_{4} \mathrm{ss}$ en $\mathrm{t}-\mathrm{ZrO}_{2}$ ss y $\mathrm{ZrTi}_{2} \mathrm{O}_{6}$.

La presencia de la superestructura en $\mathrm{ZrTi}_{2} \mathrm{O}_{6}$ indica que es un compuesto ordenado, por lo que la transición de la estructura ordenada $\left(\mathrm{ZrTi}_{2} \mathrm{O}_{6}\right)$ a la desordenada $\left(\mathrm{ZrTiO}_{4}\right)$ es de tipo orden-desorden. Con objeto de estudiar en detalle esta transformación, McHale y Roth [4, 37] prepararon muestras con composiciones binarias $\mathrm{ZrO}_{2}-\mathrm{TiO}_{2}$ con una pequeña adición de $\mathrm{Y}_{2} \mathrm{O}_{3}(0.5 \% \mathrm{~mol})$, compuesto que acelera la cinética de transformación. Sin embargo, las fases presentes a temperatura ambiente en estas muestras fueron $\mathrm{Zr}_{5} \mathrm{Ti}_{7} \mathrm{O}_{24^{\prime}}$ c- $\mathrm{ZrO}_{2}$ y el compuesto con estructura pirocloro $\mathrm{Y}_{2} \mathrm{Ti}_{2} \mathrm{O}_{7}[4]$. En el diagrama propuesto a partir de los datos obtenidos (Fig. 5), se propone el compuesto $\mathrm{Zr}_{5} \mathrm{Ti}_{7} \mathrm{O}_{24}$ como la fase estable en equilibrio con $\mathrm{TiO}_{2}$ ss a alta temperatura $\left(\approx 1200-1760^{\circ} \mathrm{C}\right)$ en composiciones ricas en $\mathrm{TiO}_{2}(>58.04 \% \mathrm{~mol})$. La fase $\mathrm{Zr}_{5} \mathrm{Ti}_{7} \mathrm{O}_{24}$ está en equilibrio con $\mathrm{ZrTi}_{2} \mathrm{O}_{6} \mathrm{y} \mathrm{TiO}_{2}$ ss en el punto peritectoide que hay a $1200^{\circ} \mathrm{C}$ en el diagrama propuesto por estos autores (Fig. 5).

Como se desprende de la discusión anterior, los trabajos de McHale y Roth $[4,37]$ pusieron de manifiesto la importancia que tienen la velocidad de enfriamiento y las impurezas en el desarrollo de fases dentro del sistema $\mathrm{ZrO}_{2}-\mathrm{TiO}_{2}$. Asimismo, se desprende el interés que presenta el estudio del sistema ternario $\mathrm{ZrO}_{2}-\mathrm{TiO}_{2}-\mathrm{Y}_{2} \mathrm{O}_{3}$.

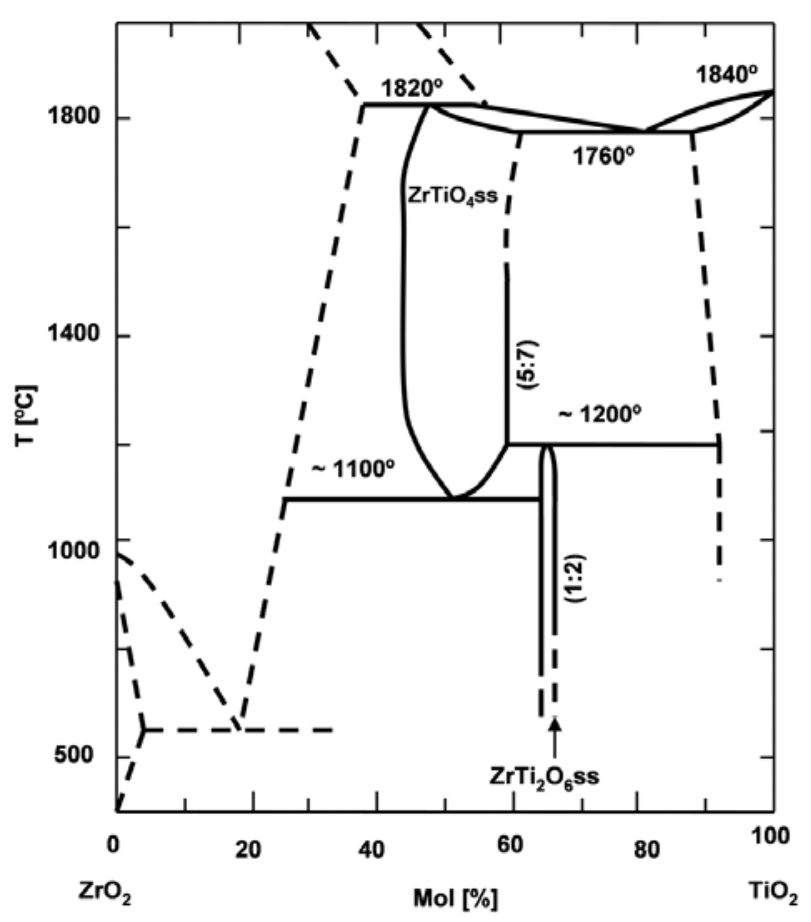

Figura 5. Diagrama de equilibrio de fases del sistema $\mathrm{ZrO}_{2}-\mathrm{TiO}_{2}$ publicado por McHale y Roth [4].
De acuerdo con el diagrama propuesto por McHale y Roth [4, 37] (Fig. 5), entre 1100 y $1200^{\circ} \mathrm{C}$ habría una región de coexistencia de las fases $\mathrm{ZrTiO}_{4} \mathrm{ss}$ y $\mathrm{ZrTi}_{2} \mathrm{O}_{6} \mathrm{ss}$ para composiciones $\approx 50-66 \% \mathrm{~mol} \mathrm{TiO}_{2}$. Sin embargo, los resultados de Troitzsch y Ellis [39], en el año 2005, no mostraron evidencias de la existencia de esta región, por lo que propusieron una modificación del diagrama entre 1000 y $1200^{\circ} \mathrm{C}$ (Fig. 6), con la presencia de dos fases ordenadas. En este diagrama, la fase $\mathrm{ZrTiO}_{4}$ ss (una de las fases $(\mathrm{Zr}, \mathrm{Ti})_{2} \mathrm{O}_{4}$ ordenada de acuerdo con la nomenclatura de los autores [39]) tendría un margen de composición muy estrecho y sería la fase estable entre $1160^{\circ} \mathrm{C}$ y $1080^{\circ} \mathrm{C}$. Por debajo de $1080^{\circ} \mathrm{C}$ la fase estable sería $\mathrm{ZrTi}_{2} \mathrm{O}_{6} \mathrm{ss}\left((\mathrm{Zr}, \mathrm{Ti})_{2} \mathrm{O}_{4}\right.$ ordenada de acuerdo con la nomenclatura de los autores [39]). La transición entre $\mathrm{ZrTiO}_{4}$ ss desordenado y ordenado viene acompañada por una reducción significativa de la longitud del eje b.

\section{SISTEMA $\mathrm{ZrO}_{2}-\mathrm{TiO}_{2}-\mathrm{Y}_{2} \mathrm{O}_{3}$}

El sistema $\mathrm{ZrO}_{2}-\mathrm{TiO}_{2}-\mathrm{Y}_{2} \mathrm{O}_{3}$ tiene importancia en la obtención de materiales de titanato de circonio-circona, puesto que el $\mathrm{Y}_{2} \mathrm{O}_{3}$ es uno de los aditivos más usados para estabilizar las fases $\mathrm{t}-\mathrm{ZrO}_{2}$ y c- $\mathrm{ZrO}_{2}$ a temperatura ambiente. Sin embargo este sistema ha recibido poca atención y no se han establecido diagramas de fases completos. En la discusión que se realiza de las figuras 8,9 y 10, se ha mantenido la nomenclatura usada por los autores en sus trabajos para representar este sistema ternario, $\mathrm{ZrO}_{2}-\mathrm{TiO}_{2}-\mathrm{YO}_{1.5^{\prime}}$ indicando entre paréntesis la conversión a la nomenclatura no usada, $\mathrm{ZrO}_{2}-\mathrm{TiO}_{2}-\mathrm{Y}_{2} \mathrm{O}_{3^{\prime}}$ con objeto de poder comparar los datos publicados en los distintos trabajos que se describen a continuación.

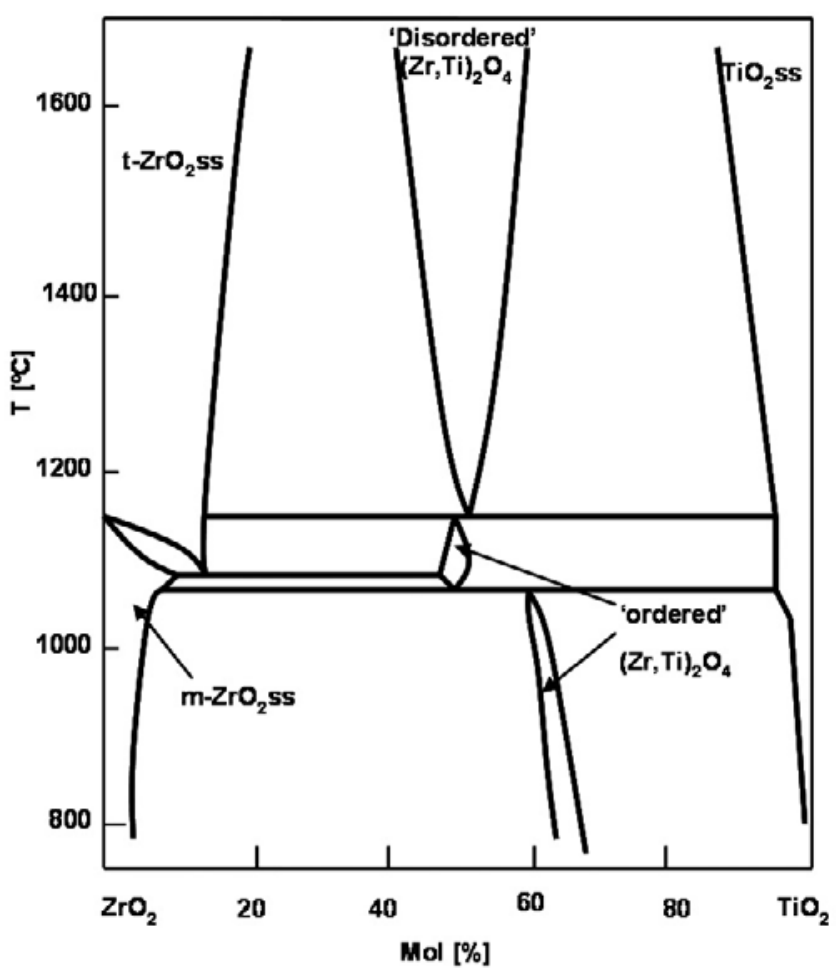

Figura 6. Diagrama de equilibrio de fases del sistema $\mathrm{ZrO}_{2}-\mathrm{TiO}_{2}$ publicado por Troitzsch y Ellis [39]. 
Uno de los primeros estudios realizados en este sistema, lo llevó a cabo Tsukuma en 1986 [40]. Este autor fabricó materiales de $\mathrm{c}-\mathrm{ZrO}_{2}$ estabilizada con $8 \%$ mol de $\mathrm{Y}_{2} \mathrm{O}_{3}$ con $\mathrm{y}$ sin adición de $10 \%$ mol de $\mathrm{TiO}_{2}$. Los materiales con $\mathrm{TiO}_{2}$ tenían un tamaño de grano mayor que los materiales $\sin \mathrm{TiO}_{2}$ y eran transparentes. Los resultados de este autor demuestran que las soluciones sólidas de $\mathrm{c}-\mathrm{ZrO}_{2}$ con $8 \%$ mol de $\mathrm{Y}_{2} \mathrm{O}_{3}$ y $10 \%$ mol de $\mathrm{TiO}_{2}$ son viables.

Posteriormente, en 1990 Lin y col. [41] estudiaron la solubilidad a $1600^{\circ} \mathrm{C}$ de $\mathrm{TiO}_{2}$ en $\mathrm{t}-\mathrm{ZrO}_{2}$, estabilizada con $3 \%$ mol de $\mathrm{Y}_{2} \mathrm{O}_{3}$, y en c- $\mathrm{ZrO}_{2}$, estabilizada con $6 \%$ mol de $\mathrm{Y}_{2} \mathrm{O}_{3}$. A partir de la determinación de la evolución de los parámetros de red con la adición de $\mathrm{TiO}_{2^{\prime}}$ concluyeron que a $1600^{\circ} \mathrm{C}$ la máxima solubilidad de $\mathrm{TiO}_{2}$ en $\mathrm{t}-\mathrm{ZrO}_{2}$ era $\approx 14-16 \%$ mol, y en $\mathrm{c}-\mathrm{ZrO}_{2} \approx 18 \% \mathrm{~mol}$. Estos autores observaron que la solución sólida de $\mathrm{TiO}_{2}$ estabiliza la fase $\mathrm{t}-\mathrm{ZrO}_{2^{\prime}}$ disminuyendo la temperatura de transformación a $\mathrm{m}-\mathrm{ZrO}_{2}$. Por el contrario, a medida que aumenta la proporción de $\mathrm{TiO}_{2}$ en $\mathrm{c}-\mathrm{ZrO}_{2}$, ésta se desestabiliza transformándose en $\mathrm{t}-\mathrm{ZrO}_{2}$. En ambos casos la disolución de $\mathrm{TiO}_{2}$ incrementa el tamaño de grano, y en el caso de la $\mathrm{t}-\mathrm{ZrO}_{2}$ se consigue un tamaño de grano de hasta $10 \mu \mathrm{m}$ sin que transforme a $\mathrm{m}-\mathrm{ZrO}_{2}$ en el enfriamiento hasta temperatura ambiente. Además, mediante difracción de rayos $\mathrm{X}$ estos autores detectaron la presencia de $\mathrm{ZrTiO}_{4}$ en materiales con concentraciones de $\mathrm{TiO}_{2}$ superiores a $20 \%$ mol.

En 1992 Pyda y col. [42] prepararon materiales de $\mathrm{ZrO}_{2}$ con contenidos de $\mathrm{TiO}_{2}$ e $\mathrm{Y}_{2} \mathrm{O}_{3}$ que variaban entre $0-28 \%$ y $0.5-3 \%$ mol, respectivamente, con objeto de obtener materiales de $\mathrm{t}-\mathrm{ZrO}_{2}$ de alta tenacidad. No encontraron $\mathrm{c}-\mathrm{ZrO}_{2}$ en ninguna de las composiciones estudiadas. En los materiales con contenidos en $\mathrm{Y}_{2} \mathrm{O}_{3}$ entre $1.5-3 \%$ mol, la fase mayoritaria fue $\mathrm{t}-\mathrm{ZrO}_{2}$. A pesar de que la temperatura de sinterización usada por estos autores es muy inferior a la usada por Lin y col. [41], los valores de solución sólida de $\mathrm{TiO}_{2}$ publicados por Pyda y col. [42] son muy superiores. Así, describen soluciones sólidas de $28 \%$ mol de $\mathrm{TiO}_{2}$ en $\mathrm{ZrO}_{2}$ con $1.5 \%$ mol de $\mathrm{Y}_{2} \mathrm{O}_{3}$.

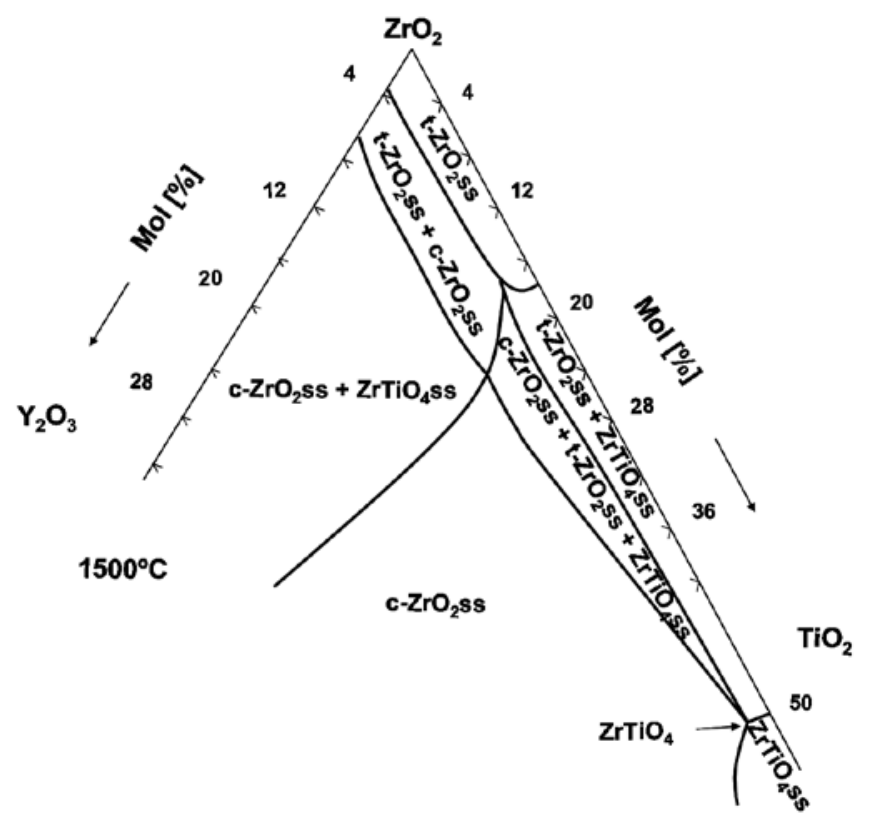

Figura 7. Sección isotermal a $1500^{\circ} \mathrm{C}$ del diagrama de equilibrio de fases del sistema $\mathrm{ZrO}_{2}-\mathrm{TiO}_{2}-\mathrm{Y}_{2} \mathrm{O}_{3}$ propuesto por Colomer y col. [46].
Estos autores atribuyen estas diferencias a que su método de síntesis por coprecipitación y posterior tratamiento térmico a $1300^{\circ} \mathrm{C}$ permite obtener materiales con mayor homogeneidad composicional que el método de reacción en estado sólido usado por Lin y col. [41]. Además, Pyda y col. [42] detectaron la formación de $\mathrm{ZrTiO}_{4}$ en materiales con un $28 \%$ mol de $\mathrm{TiO}_{2}$ y $0.5-1 \%$ mol de $\mathrm{Y}_{2} \mathrm{O}_{3}$.

En 1993 Yokokawa y col. [43] establecieron una sección isotermal teórica a $1300^{\circ} \mathrm{C}$ del sistema $\mathrm{ZrO}_{2}-\mathrm{TiO}_{2}-\mathrm{Y}_{2} \mathrm{O}_{3}$ basándose en datos termodinámicos disponibles en la bibliografía para el $\mathrm{ZrO}_{2}$ y calculando los de los otros óxidos a partir de correlaciones entre parámetros de interacción y radios iónicos. Calcularon que a $1300^{\circ} \mathrm{C}$ la solubilidad de $\mathrm{TiO}_{2}$ en $\mathrm{ZrO}_{2}$ con $8 \%$ mol de $\mathrm{Y}_{2} \mathrm{O}_{3}$ era aproximadamente $15 \%$ mol. Este valor de solubilidad calculado teóricamente por estos autores, concuerda con los datos experimentales publicados por Liou y Worrel a $1600^{\circ} \mathrm{C}$ [44]. Además, Yokokawa y col. [45] propusieron que, aunque en el sistema $\mathrm{ZrO}_{2}-\mathrm{Y}_{2} \mathrm{O}_{3}$ no hay posibilidad de existencia de fases con estructura pirocloro, es posible la existencia de soluciones sólidas de fórmula $\mathrm{Y}_{2}(\mathrm{Ti}, \mathrm{Zr})_{2} \mathrm{O}_{7}$ en el sistema $\mathrm{ZrO}_{2}-\mathrm{TiO}_{2}-\mathrm{Y}_{2} \mathrm{O}_{3}$, sugiriendo que hay un rango de composición donde coexisten $\mathrm{Y}_{2}(\mathrm{Ti}, \mathrm{Zr})_{2} \mathrm{O}_{7} \mathrm{ss}$ y $\mathrm{c}-\mathrm{ZrO}_{2} \mathrm{ss}$.

Más tarde, en 1997, Colomer y col. [46] establecieron una sección isotermal experimental a $1500^{\circ} \mathrm{C}$ centrándose en la zona rica en $\mathrm{ZrO}_{2}(65-97 \%$ mol$)$ que muestra la figura 7. De acuerdo con estos autores, a $1500^{\circ} \mathrm{C}$ el $\mathrm{ZrTiO}_{4}$ ss puede coexistir con $\mathrm{t}-\mathrm{ZrO}_{2} \mathrm{ss}$ y c- $\mathrm{ZrO}_{2}$ ss dependiendo de la cantidad de $\mathrm{Y}_{2} \mathrm{O}_{3}$. Para contenidos de $\mathrm{Y}_{2} \mathrm{O}_{3}$ menores de $3 \%$ mol y de $\mathrm{TiO}_{2}$ inferiores a $17.9 \%$ mol la única fase en equilibrio es $\mathrm{t}-\mathrm{ZrO}_{2} \mathrm{ss}$, mientras que para cantidades de $\mathrm{TiO}_{2}$ superiores a $17.9 \%$ mol coexisten $\mathrm{t}-\mathrm{ZrO}_{2} \mathrm{ss}$ y $\mathrm{ZrTiO}_{4}$ ss. Para contenidos de $\mathrm{Y}_{2} \mathrm{O}_{3}$ superiores a $3 \% \mathrm{~mol}$, aparece $\mathrm{c}-\mathrm{ZrO}_{2}$ ss para contenidos de $\mathrm{TiO}_{2}$ entre $0-50 \%$ mol. Para contenidos en $\mathrm{Y}_{2} \mathrm{O}_{3}$ superiores a $6.6 \%$ mol no hay presencia de $\mathrm{t}-\mathrm{ZrO}_{2} \mathrm{ss}$. A medida que aumenta la cantidad de $\mathrm{Y}_{2} \mathrm{O}_{3^{\prime}}$ la cantidad de $\mathrm{TiO}_{2}$ necesaria para formar

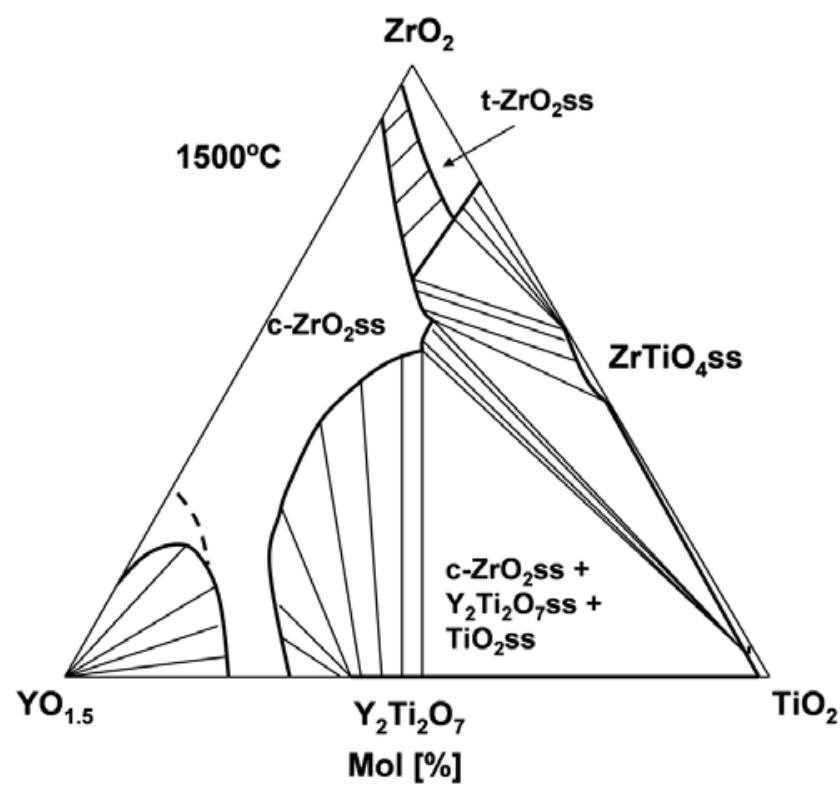

Figura 8. Sección isotermal a $1500^{\circ} \mathrm{C}$ del diagrama de equilibrio de fases del sistema $\mathrm{ZrO}_{2}-\mathrm{TiO}_{2}-\mathrm{Y}_{2} \mathrm{O}_{3}$ propuesto por Kobayashi y col. [48]. La región rica en circona coincide con la propuesta por Colomer y col. [46]. 
$\mathrm{ZrTiO}_{4}$ ss se incrementa hasta un máximo de $\approx 26-28 \% \mathrm{~mol}$

En ese mismo año (1997), Traqueia y col. [47] publicaron un estudio sobre la conductividad iónica en materiales de c- $\mathrm{ZrO}_{2}$ con $8 \%$ mol de $\mathrm{Y}_{2} \mathrm{O}_{3}$ a los que le añadían cantidades crecientes de $\mathrm{TiO}_{2}$ hasta un máximo de $20 \%$ mol. Pusieron de manifiesto que en los materiales con $20 \%$ mol de $\mathrm{TiO}_{2}$ se formaba $\mathrm{ZrTiO}_{4}$. Además, concluyeron que la adición de $\mathrm{TiO}_{2}$ disminuía la conductividad iónica de $\mathrm{c}-\mathrm{ZrO}_{2}$.

En 1998, Kobayashi y col. [48] establecieron experimentalmente secciones isotermales del sistema $\mathrm{ZrO}_{2}$ $\mathrm{TiO}_{2}-\mathrm{Y}_{2} \mathrm{O}_{3}$ a distintas temperaturas. La figura 8 muestra la sección isotermal completa a $1500^{\circ} \mathrm{C}$. Estudiaron distintas composiciones dentro de los sistemas binarios $\mathrm{ZrO}_{2}-\mathrm{TiO}_{2^{\prime}}$ $\mathrm{ZrO}_{2}-\mathrm{YO}_{1.5}$ y TiO ${ }_{2}-\mathrm{YO}_{1.5}$ y del sistema ternario $\mathrm{ZrO}_{2}-\mathrm{TiO}_{2}-\mathrm{YO}_{1.5}$. Estos autores propusieron un campo monofásico de $\mathrm{ZrTiO}_{4} \mathrm{ss}$ con un límite de solubilidad de $Y \mathrm{Y}_{1.5} \mathrm{de} \approx 1.0 \% \mathrm{~mol}(\approx 0.5 \% \mathrm{~mol}$ $\mathrm{Y}_{2} \mathrm{O}_{3}$. Valor aproximado calculado a partir de la representación gráfica incluida en la referencia [48]). Es de señalar que esta región monofásica fue propuesta en tentativa, a partir de datos experimentales del sistema $\mathrm{ZrO}-\mathrm{TiO}_{2}$, pero sin puntos experimentales correspondientes a composiciones ternarias con concentraciones de $\mathrm{YO}_{1.5}$ en torno a $1 \% \mathrm{~mol}(\approx 0.5 \% \mathrm{~mol}$ $\mathrm{Y}_{2} \mathrm{O}_{3}$ ). La composición experimental más cercana a la zona de $1 \%$ mol de $\mathrm{YO}_{1.5}$ estudiada por estos autores, está constituida por $\approx 46 \%$ mol de $\mathrm{ZrO}_{2^{\prime}} \approx 46 \% \mathrm{~mol} \mathrm{de} \mathrm{TiO}_{2} \mathrm{y} \approx 8 \%$ mol de $\mathrm{YO}_{1.5}$ $\left(\approx 48 \% \mathrm{~mol} \mathrm{de} \mathrm{ZrO}_{2} \approx 48 \% \mathrm{~mol} \mathrm{de} \mathrm{TiO}_{2} \mathrm{y} \approx 4 \%\right.$ mol de $\mathrm{Y}_{2} \mathrm{O}_{3}$ ).

En 1999, Feighery y col. [49] establecieron experimentalmente una sección isotermal completa a $1500^{\circ} \mathrm{C}$ (Fig.9) donde se observa una región monofásica de $\mathrm{ZrTiO}_{4}$ ss en el rango de concentraciones de $\mathrm{ZrO}_{2}$ comprendido entre 40-55\% mol. Esto concuerda con los datos de McHale y Roth [4, 37] (Fig. 5). Se puede observar cómo en esta sección isotermal (Fig. 9) $\mathrm{ZrTiO}_{4} \mathrm{ss}$ coexiste con $\mathrm{Y}_{2} \mathrm{Ti}_{2} \mathrm{O}_{7}$ ss. Además, el límite de solubilidad de $\mathrm{YO}_{1.5}$ en la región monofásica de $\mathrm{ZrTiO}_{4} \mathrm{ss}$ es $\approx 2 \% \mathrm{~mol}(\approx 1 \% \mathrm{~mol}$ $\mathrm{Y}_{2} \mathrm{O}_{3}$. Valor aproximado calculado a partir de la representación gráfica incluida en la referencia [49]). Al igual que Kobayashi y

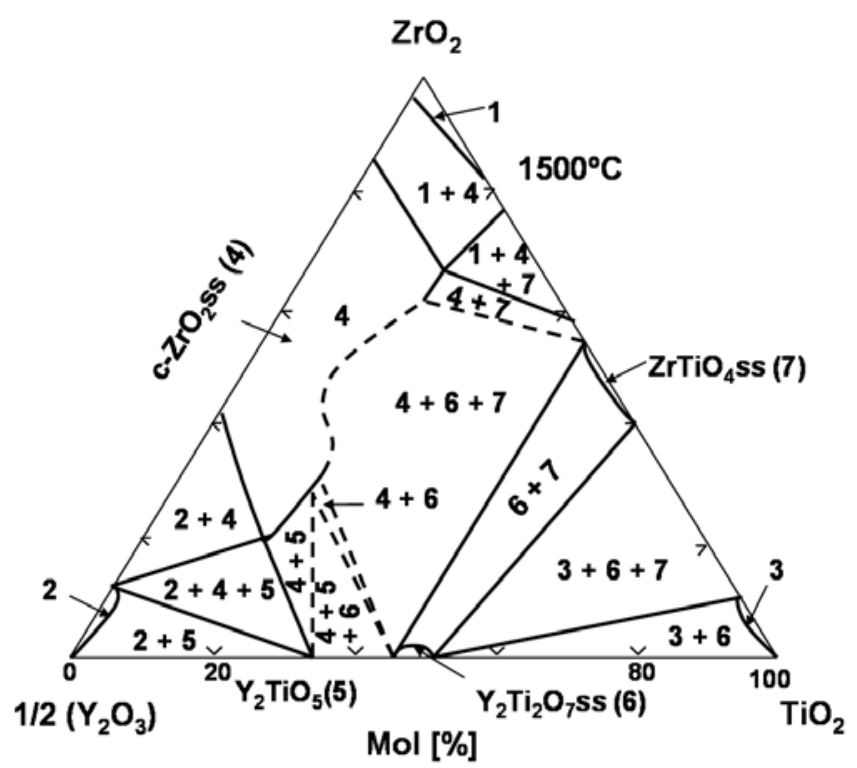

Figura 9. Sección isotermal a $1500^{\circ} \mathrm{C}$ del diagrama de equilibrio de fases del sistema $\mathrm{ZrO}_{2}-\mathrm{TiO}_{2}-\mathrm{Y}_{2} \mathrm{O}_{3}$ propuesto por Feighery y col. [49]. col. [48], Feighery y col. [49] proponen este límite de solubilidad en tentativa ya que tampoco se basan en datos experimentales. La composición experimental más cercana a esa región es $\approx 54 \%$ $\mathrm{mol} \mathrm{ZrO} 2^{\prime} \approx 43 \% \mathrm{~mol} \mathrm{TiO}_{2} \mathrm{y} \approx 3 \% \mathrm{~mol} \mathrm{YO} \mathrm{YO}_{1.5}\left(\approx 54.5 \% \mathrm{~mol} \mathrm{ZrO}_{2^{\prime}}\right.$ $\approx 44.0 \% \mathrm{~mol} \mathrm{TiO}_{2} \mathrm{y} \approx 1.5 \% \mathrm{~mol} \mathrm{Y}_{2} \mathrm{O}_{3}$ ).

En 2008, Schaedler y col. [50] establecieron secciones isotermales a 1300, 1500 y $1600^{\circ} \mathrm{C}$ combinando datos experimentales y trabajo de modelización. La figura 10 muestra la sección isotermal propuesta por estos autores a $1500^{\circ} \mathrm{C}$. Se pueden observar diferencias significativas en cuanto a la compatibilidad de fases propuesta por Feighery y col. [49]. En concreto, en la sección isotermal propuesta por Schaedler y col. a $1500^{\circ} \mathrm{C}$ [50] (Fig.10) hay compatibilidad entre las fases $\mathrm{ZrTiO}_{4}$ ss y c-ZrO $\mathrm{Zrs}_{2}$. La compatibilidad propuesta por Feighery y col. [49] (Fig. 9) a $1500^{\circ} \mathrm{C}$ entre $\mathrm{Y}_{2} \mathrm{Ti}_{2} \mathrm{O}_{7}$ ss y $\mathrm{ZrTiO}_{4}$ ss también la proponen Schaedler y col. [50] pero a $1300^{\circ} \mathrm{C}$. Al igual que Feighery y col. [49], Schaedler y col. [50] proponen el mismo límite de solubilidad de $\mathrm{YO}_{1.5^{\prime}} \approx 2 \% \mathrm{~mol}(\approx 1 \% \mathrm{~mol}$ $\mathrm{Y}_{2} \mathrm{O}_{3}$ ), en la región monofásica de $\mathrm{ZrTiO}_{4} \mathrm{ss}$ (límite calculado de manera aproximada a partir de la representación gráfica incluida en la referencia [50]). La composición experimental estudiada por Schaedler y col. [50] más cercana a la región monofásica de $\mathrm{ZrTiO}_{4}$ ss es $40 \%$ mol $\mathrm{ZrO}_{2}, 50 \% \mathrm{~mol} \mathrm{TiO}_{2}$ y $10 \%$

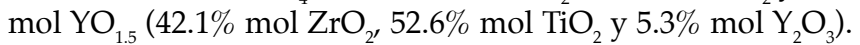

Tal y como se ha discutido, las secciones isotermales del sistema $\mathrm{ZrO}_{2}-\mathrm{TiO}_{2}-\mathrm{YO}_{1.5}$ propuestas a $1500^{\circ} \mathrm{C}$ por Kobayashi y col. [48], Feighery y col. [49] y Schaedler y col. [50] (Fig. 8, 9 y 10) presentan diferencias en cuanto a compatibilidad de fases y a los límites de las distintas regiones. Sin embargo, las tres secciones isotermales presentan en tentativa una región monofásica de $\mathrm{ZrTiO}_{4}$ ss situada aproximadamente entre 40-55\% mol de $\mathrm{ZrO}_{2}$ en la línea binaria $\mathrm{ZrO}_{2}-\mathrm{TiO}_{2}$. Kobayashi y col. [48] propusieron en tentativa un límite de solubilidad de $\mathrm{YO}_{1.5}$ en $\mathrm{ZrTiO}_{4}$ ss de $\approx 1 \% \mathrm{~mol}\left(\approx 0.5 \%\right.$ mol $\left._{2} \mathrm{O}_{3}\right)$, mientras que Feighery y col. [49] y Schaedler y col. [50] establecieron, también en tentativa, ese límite de solubilidad de $\mathrm{YO}_{1.5}$ en

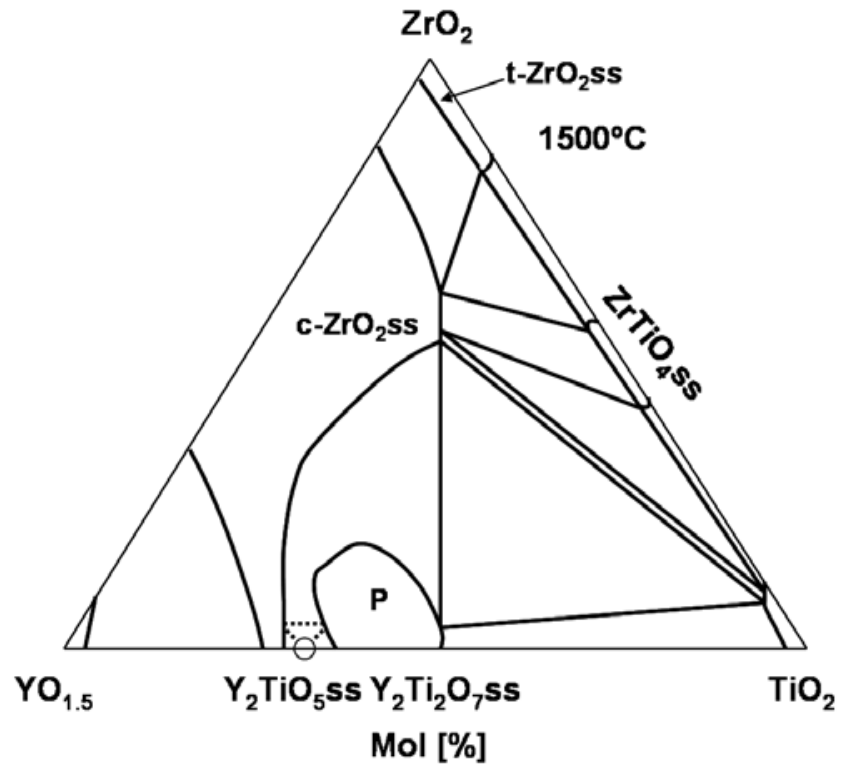

Figura 10. Sección isotermal a $1500^{\circ} \mathrm{C}$ del diagrama de equilibrio de fases del sistema $\mathrm{ZrO}_{2}-\mathrm{TiO}_{2}-\mathrm{Y}_{2} \mathrm{O}_{3}$ propuesto por Schaedler y col. [50]. Este diagrama de equilibrio coincide en su práctica totalidad con el propuesto por Kobayashi y col. [48]. 
$\mathrm{ZrTiO}_{4}$ ss en $\approx 2 \%$ mol $\left(\approx 1 \%\right.$ mol $\left.\mathrm{Y}_{2} \mathrm{O}_{3}\right)$. Ninguno de estos autores determinó experimentalmente el límite de solubilidad de $\mathrm{YO}_{1.5}$ en $\mathrm{ZrTiO}_{4}$ ss. Es lógico pensar que Kobayashi y col. [48], Feighery y col. [49] y Schaedler y col. [50] propusieran los límites de solubilidad de $\mathrm{YO}_{1.5}$ en $\mathrm{ZrTiO}_{4}$ ss basándose en el trabajo experimental de McHale y Roth [4]. Estos autores encontraron $\mathrm{ZrTiO}_{4}$ como única fase presente en materiales preparados a $1500^{\circ} \mathrm{C}$ a partir de mezclas de $\mathrm{ZrO}_{2}$ y $\mathrm{TiO}_{2}$, cuya composición variaba entre 41.46-54.73\% mol de $\mathrm{ZrO}_{2}$ y 58.04$44.77 \% \mathrm{~mol}$ de $\mathrm{TiO}_{2}$, con una cantidad fija de $0.5 \% \mathrm{~mol}$ de $\mathrm{Y}_{2} \mathrm{O}_{3}\left(1 \%\right.$ mol $\left.\mathrm{YO}_{15}\right)$ en todos los casos. En este mismo trabajo, McHale y Roth [4] proponen que el límite de solubilidad de $\mathrm{Y}_{2} \mathrm{O}_{3}$ en $\mathrm{ZrTiO}_{4}$ ss a $1500^{\circ} \mathrm{C}$ está comprendido entre $0.5-1.0 \%$ mol (1-2\% mol $\mathrm{YO}_{1.5}$ ). Dado que estos autores no estudiaron composiciones con adiciones de $\mathrm{Y}_{2} \mathrm{O}_{3}$ distintas a $0.5 \%$ en mol $\left(1 \%\right.$ mol $\left.\mathrm{YO}_{1.5}\right)$ [4], no hay evidencia experimental de que no haya soluciones sólidas a $1500^{\circ} \mathrm{C}$ de $\mathrm{ZrTiO}_{4}$ ss con contenidos superiores a $1 \% \mathrm{~mol}$ de $\mathrm{Y}_{2} \mathrm{O}_{3}\left(2 \% \mathrm{~mol} \mathrm{YO}_{1.5}\right)$.

Como señalan Fagg y col. [51] las composiciones dentro del sistema $\mathrm{ZrO}_{2}-\mathrm{TiO}_{2}-\mathrm{Y}_{2} \mathrm{O}_{3}$ son muy sensibles a pequeñas variaciones de temperatura, composición y velocidades de enfriamiento. Por lo tanto, las discrepancias existentes pueden deberse a las distintas condiciones experimentales utilizadas. En todos los trabajos descritos se utilizó la reacción en estado sólido a partir de polvos como método de fabricación de las probetas. Sin embargo, Kobayashi y col. [48] utilizaron polvos obtenidos vía sol-gel a partir de mezclas de alcóxidos con un posterior tratamiento térmico a $1500^{\circ} \mathrm{C}$ durante $10 \mathrm{~h}$. Feighery y col. [49] prepararon materiales a $1500^{\circ} \mathrm{C}$ durante $36 \mathrm{~h}$ a partir de polvos de alta pureza de $\mathrm{Y}_{2} \mathrm{O}_{3^{\prime}}, \mathrm{ZrO}_{2}$ y TiO ${ }_{2}$, con moliendas intermedias y enfriamientos bruscos. Schaedler y col. [50] usaron métodos de coprecipitación para la obtención de los polvos y realizaron tratamientos térmicos a $1500^{\circ} \mathrm{C}$ durante 50-220h. Las velocidades de calentamiento y enfriamiento eran de $10^{\circ} \mathrm{C} / \mathrm{min}$ en la mayoría de las composiciones estudiadas.

En principio, los métodos sol-gel y de coprecipitación dan lugar a mezclas más íntimas de los componentes que la mezcla de óxidos. Por otra parte, la molienda intermedia hace que nuevas superficies entren en contacto durante el prensado, por lo que favorece que la reacción prosiga. Además, para retener las fases de alta temperatura es preciso congelar los equilibrios alcanzados a elevadas temperaturas (mediante enfriamientos bruscos), como se hace de manera sistemática en la elaboración

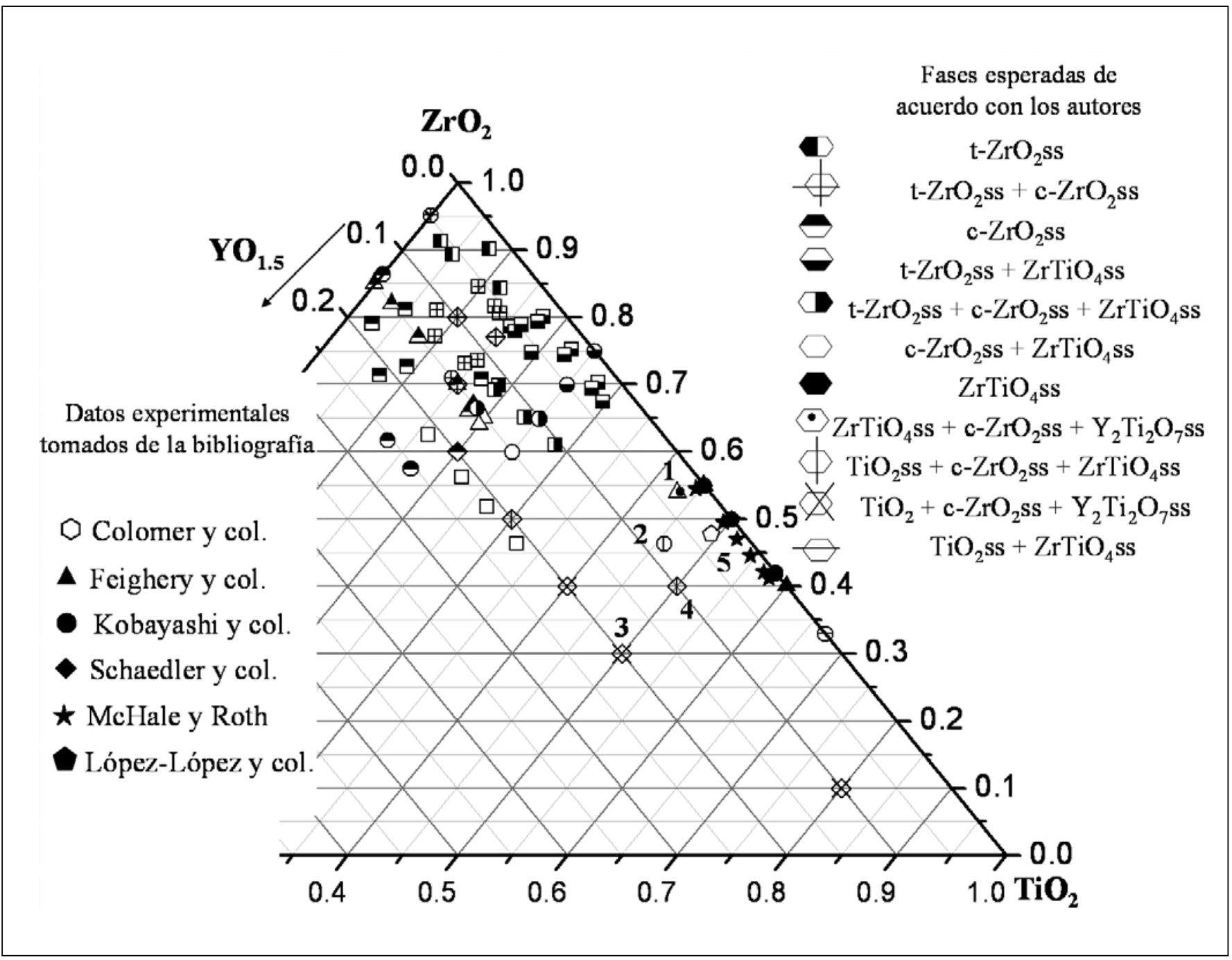

Figura 11. Sistema ternario $\mathrm{ZrO}_{2}-\mathrm{TiO}_{2}-\mathrm{Y}_{2} \mathrm{O}_{3}$ donde se señalan los puntos experimentales publicados en bibliografía por distintos autores. Las fases propuestas por Feighery y col. [49] para el punto experimental 1 no están de acuerdo con las fases propuestas por Kobayashi y col. [48], Schaedler y col. [50] y López-López y col. [31] para los puntos experimentales 2, 3, 4 y 5, respectivamente. 
de diagramas de equilibrio de fases, porque, tal y como se ha discutido anteriormente, la velocidad de enfriamiento es fundamental en la evolución de las composiciones estudiadas en este sistema.

En 2010 López-López y col. [31] estudiaron mezclas equimolares de $\mathrm{TiO}_{2}$ y $\mathrm{t}-\mathrm{ZrO}_{2}$ estabilizada con $3 \%$ mol de $\mathrm{Y}_{2} \mathrm{O}_{3}$ calentadas a $1500^{\circ} \mathrm{C}$ durante 2 y 30 horas con velocidades de calentamiento y enfriamiento de $5^{\circ} \mathrm{C} / \mathrm{min}$. Encontraron un compuesto con estructura pirocloro en los bordes de grano de $\mathrm{ZrTiO}_{4}$ y c- $\mathrm{ZrO}_{2} \mathrm{ss}$ en los materiales tratados durante 2 horas. Los tratamientos a 30 horas demostraron que este compuesto no es una fase de equilibro a $1500^{\circ} \mathrm{C}$. Una explicación plausible es que el compuesto de tipo pirocloro se forma a baja temperatura debido a su menor energía libre de formación $\left(-4593 \mathrm{~kJ} / \mathrm{mol}\right.$ a $1000^{\circ} \mathrm{C}$ para el compuesto con estructura pirocloro y $-2292 \mathrm{~kJ} / \mathrm{mol}$ a $1000^{\circ} \mathrm{C}$ para $\mathrm{ZrTiO}_{4^{\prime}}$ calculadas usando las ecuaciones publicadas por Schaedler y col. [50]) y a alta temperatura desaparece en función del tiempo de permanencia. La no estabilidad del compuesto con estructura pirocloro está en desacuerdo con la sección isotermal propuesta por Feighery y col. a $1500^{\circ} \mathrm{C}$ [49].

$\mathrm{Si}$ se representan en un diagrama ternario los puntos experimentales estudiados por McHale y Roth [4], Colomer y col.[46], Kobayashi y col. [48], Feighery y col. [49], Schaedler y col. [50] y López-López y col. [31] (Fig. 11. Se ha usado la nomenclatura $\mathrm{ZrO}_{2}-\mathrm{TiO}_{2}-\mathrm{YO}_{1.5}$ para representar todas las composiciones) indicando las fases encontradas por los autores para las distintas composiciones representadas, se pueden observar algunas discrepancias en los límites de compatibilidad de las distintas regiones. La discrepancia más clara se puede observar en que el punto experimental de composición $\approx 53.9 \% \mathrm{~mol} \mathrm{ZrO}_{2^{\prime}} \approx 43.1 \% \mathrm{~mol} \mathrm{TiO}_{2^{\prime}} \approx 3.0 \% \mathrm{~mol}$ $\mathrm{YO}_{1.5}$ (punto 1 en la fig. 11), el cual según Feighery y col. [49] estaría situado en una región trifásica de $\mathrm{ZrTiO}_{4} \mathrm{ss}, \mathrm{c}-\mathrm{ZrO}_{2} \mathrm{ss}$ e $\mathrm{Y}_{2} \mathrm{Ti}_{2} \mathrm{O}_{7} \mathrm{ss}$, sería incompatible con los puntos estudiados por

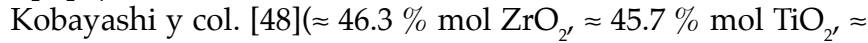
$8.0 \% \mathrm{~mol} \mathrm{YO}_{1.5^{\prime}}$ punto 2 en la fig. 11) y Schaedler y col. [50] (30 $\% \mathrm{~mol} \mathrm{ZrO}_{2}, 50 \% \mathrm{~mol} \mathrm{TiO}{ }_{2}, 20 \% \mathrm{~mol} \mathrm{YO} \mathrm{YO}_{1.5}$ y $40 \% \mathrm{~mol} \mathrm{Z \textrm {ZO } _ { 2 }}$ $50 \% \mathrm{~mol} \mathrm{TiO}_{2^{\prime}} 10 \% \mathrm{~mol} \mathrm{YO}_{1.5^{\prime}}$ puntos 3 y 4, respectivamente en la fig. 11) que estarían en una región trifásica de $\mathrm{ZrTiO}_{4} \mathrm{ss}$, c- $\mathrm{ZrO}_{2} \mathrm{ss}$ y $\mathrm{TiO}_{2} \mathrm{ss}$, y con el punto experimental estudiado por López-López y col. [31] (47.78 \% mol $\mathrm{ZrO}_{2^{\prime}} 49.26 \% \mathrm{~mol} \mathrm{TiO}_{2^{\prime}}$ $2.96 \%$ mol $\mathrm{YO}_{1.5^{\prime}}$ punto 5 en la fig. 11) el cual estaría en una región bifásica de $\mathrm{ZrTiO}_{4}$ ss y c- $\mathrm{ZrO}_{2} \mathrm{ss}$.

\section{EXPANSIÓN TÉRMICA DEL TITANATO DE CIRCONIO}

La expansión térmica de los materiales cerámicos determina las tensiones que se generan en un material como consecuencia de las variaciones temporales o gradientes espaciales de temperatura. Por tanto, es una propiedad fundamental a estudiar para poder diseñar materiales resistentes al choque térmico $[52,53]$.

Uno de los primeros estudios sobre la expansión térmica del titanato de circonio lo realizaron Lynch y Morosin [54] en 1972, los cuales determinaron los parámetros de red a temperatura ambiente de tres materiales monofásicos de $\mathrm{ZrTiO}_{4}$ fabricados a partir de polvos sintetizados por reacción en estado sólido de mezclas estequiométricas de polvos de $\mathrm{ZrO}_{2}$ y TiO${ }_{2}$. El material de partida se obtuvo por tratamiento térmico a $1625^{\circ} \mathrm{C}$ durante $6 \mathrm{~h}\left(1625^{\circ} \mathrm{C}-6 \mathrm{~h}\right.$, velocidad de enfriamiento no especificada) y los otros dos materiales se obtuvieron por recocido a $1300^{\circ} \mathrm{C}$ del material inicial, utilizando dos ciclos diferentes: tratamiento isotermo a $1300^{\circ} \mathrm{C}$ durante $72 \mathrm{~h}$ y enfriamiento brusco $\left(1300^{\circ} \mathrm{C}-72 \mathrm{~h}\right)$ y tratamiento isotermo a $1300^{\circ} \mathrm{C}$ durante $24 \mathrm{~h}$ y enfriamiento lento $\left(1300^{\circ} \mathrm{C}-24 \mathrm{~h}\right.$, velocidad de enfriamiento no especificada). Estos autores observaron que las longitudes del eje cristalográfico b en los materiales inicial $\left(1625^{\circ} \mathrm{C}-6 \mathrm{~h}\right)$ y enfriado lentamente desde $1300^{\circ} \mathrm{C}\left(1300^{\circ} \mathrm{C}-24 \mathrm{~h}\right)$ eran similares mientras que en el material $1300^{\circ} \mathrm{C}-72 \mathrm{~h}$ era significativamente mayor $(\Delta \mathrm{b} \approx 0.070 \AA$ ) $)$. Esta diferencia entre los ejes $b$ de materiales enfriados lenta y bruscamente es del mismo signo que la observada por Coughanour y col. [2].

Además, Lynch y Morosin, analizaron la expansión térmica del material enfriado lentamente. La tabla I muestra los coeficientes medios de expansión térmica de los ejes cristalográficos de este material $\left(\alpha_{a^{\prime}} \alpha_{b}\right.$ y $\left.\alpha_{c}\right)$, calculados entre 25 y $600^{\circ} \mathrm{C}$ a partir de la gráfica recogida en su artículo [54].

En 1988 Ikawa y col. [55] estudiaron la expansión térmica de materiales monofásicos de $\mathrm{ZrTiO}_{4}$ obtenidos a partir de mezclas estequiométricas de $\mathrm{ZrO}_{2}$ y TiO 2 obtenidas por coprecipitación. El material de partida se obtuvo por tratamiento térmico a $1600^{\circ} \mathrm{C}$ durante un tiempo no especificado entre $5-10 \mathrm{~h}$ con velocidad de enfriamiento de $10^{\circ} \mathrm{C} / \mathrm{min}\left(\mathrm{ZrTiO}_{4}-1600^{\circ} \mathrm{C}\right)$. Los otros dos materiales fueron obtenidos a partir de polvos de este material tratados a menor temperatura y realizando enfriamientos bruscos. Los tratamientos isotermos utilizados fueron $5 \mathrm{~h}$ a $1500^{\circ} \mathrm{C}\left(\mathrm{ZrTiO}_{4}-1500\right)$ y $720 \mathrm{~h}$ a $1000^{\circ} \mathrm{C}\left(\mathrm{ZrTiO}_{4}^{-}\right.$ $1000^{\circ} \mathrm{C}$ ). En la tabla I se recogen los coeficientes medios de expansión térmica entre 25 y $600^{\circ} \mathrm{C}$ de los ejes cristalográficos

TABla I. COEFICIENTES MEDIOS DE EXPANSIÓN TÉRMICA CALCUlADOS ENTRE 25-600C A PARTIR DE LA GRÁFICA Y DATOS PUBLICADOS POR LYNCH Y MOROSIN [54] E IKAWA Y COL. [55], RESPECTIVAMENTE.

\begin{tabular}{|c|c|c|c|c|c|}
\hline Material & $\begin{array}{c}\text { Tratamiento } \\
\text { Térmico }\end{array}$ & Enfriamiento & $\alpha_{\mathrm{a}}\left({ }^{\circ} \mathrm{C}^{-1}\right)$ & $\alpha_{\mathrm{b}}\left({ }^{\circ} \mathrm{C}^{-1}\right)$ & $\alpha_{\mathrm{c}}\left({ }^{\circ} \mathrm{C}^{-1}\right)$ \\
\hline $\mathrm{ZrTiO}_{4}-1000[55]$ & $1000^{\circ} \mathrm{C} / 720 \mathrm{~h}$ & Brusco & $\approx 4.3 \times 10^{-6}$ & $\approx 16.0 \times 10^{-6}$ & $\approx 7.7 \times 10^{-6}$ \\
\hline $\mathrm{ZrTiO}_{4}-1300[54]$ & $1300^{\circ} \mathrm{C} / 24 \mathrm{~h}$ & Lento & $\approx 6.2 \times 10^{-6}$ & $\approx 12.3 \times 10^{-6}$ & $\approx 7.7 \times 10^{-6}$ \\
\hline $\mathrm{ZrTiO}_{4}-1600[55]$ & $1600^{\circ} \mathrm{C} / 5-10 \mathrm{~h}$ & $10^{\circ} \mathrm{C} / \mathrm{min}$ & $\approx 6.4 \times 10^{-6}$ & $\approx 10.2 \times 10^{-6}$ & $\approx 8.1 \times 10^{-6}$ \\
\hline $\mathrm{ZrTiO}_{4}-1500[55]$ & $1500^{\circ} \mathrm{C} / 5 \mathrm{~h}$ & Brusco & $\approx 6.9 \times 10^{-6}$ & $\approx 4.9 \times 10^{-6}$ & $\approx 7.3 \times 10^{-6}$ \\
\hline
\end{tabular}


$\left(\alpha_{a^{\prime}} \alpha_{b}\right.$ y $\left.\alpha_{c}\right)$ de los tres materiales, calculados a partir de los datos publicados por estos autores [55].

Si se comparan los resultados de la tabla I, los valores de $\alpha$ correspondientes a los ejes a y c son similares para los 4 materiales. Sin embargo, existen diferencias significativas entre los valores correspondientes al eje $b$ en función del tratamiento térmico, siendo significativamente inferior para el material cuya estructura de alta temperatura ha sido congelada mediante enfriamiento brusco $\left(1500^{\circ} \mathrm{C}\right), \mathrm{y}$ aumentando con el tiempo de permanencia a temperaturas comprendidas entre $\approx$ $1000-1300^{\circ} \mathrm{C}$.

Los resultados recogidos en la tabla I están de acuerdo con las observaciones de Park y Kim [10] en materiales monofásicos de $\mathrm{ZrTiO}_{4}$ preparados a partir de polvos sintetizados por reacción en estado sólido de mezclas estequiométricas de $\mathrm{ZrO}_{2}$ y $\mathrm{TiO}_{2}$. Estos autores pusieron de manifiesto cómo a medida que aumenta la velocidad de enfriamiento $\left(0.5-100^{\circ} \mathrm{C} / \mathrm{h}\right)$ desde la temperatura de sinterización $\left(1400^{\circ} \mathrm{C}-4 \mathrm{~h}\right)$, la longitud del eje cristalográfico b (eje c según la convención usada por Park y Kim [10]) aumenta significativamente. Asimismo Park y Kim [10] observaron una expansión térmica durante calentamientos hasta $1400^{\circ} \mathrm{C}$ menor en el material enfriado a $100^{\circ} \mathrm{C} / \mathrm{h}$ que en el enfriado a $1^{\circ} \mathrm{C} / \mathrm{h}$.

Por último, Park [56] estudió la expansión térmica de los ejes cristalográficos de un monocristal de $\mathrm{ZrTiO}_{4}$ enfriado muy lentamente $\left(0.3^{\circ} \mathrm{C} / \mathrm{h}\right)$ desde $1300^{\circ} \mathrm{C}$ hasta temperatura ambiente y determinó dos variaciones bruscas en la longitud del eje b (eje c según la convención usada por Park [56]) a T $\approx 1125^{\circ} \mathrm{C}$ y a $\mathrm{T} \approx 845^{\circ} \mathrm{C}$ las cuales atribuyó a sendos cambios de fase.

\section{CONSIDERACIONES GENERALES}

De la discusión anterior se desprende que, a temperaturas superiores a $1200^{\circ} \mathrm{C}$, en el sistema binario $\mathrm{ZrO}_{2}-\mathrm{TiO}_{2}$ existe un compuesto intermedio, de composición $\mathrm{ZrTiO}_{4}$ y estructura cristalina ortorrómbica, que admite solución sólida de $\mathrm{TiO}_{2}$ y de $\mathrm{ZrO}_{2}$. Distintos autores han estudiado experimentalmente y/o propuesto en tentativa rangos de solución sólida a diferentes temperaturas. En general, los datos reportados son complementarios y no existen contradicciones significativas entre ellos. A presión atmosférica, la composición de esta fase de alta temperatura se puede representar por la fórmula $\left(\mathrm{Zr}_{\mathrm{x}} \mathrm{Ti}_{1-\mathrm{x}}\right)_{2} \mathrm{O}_{4^{\prime}}$ donde la fracción molar de $\mathrm{Ti},(1-\mathrm{x})$, varía entre $0.42-0.58$.

Si bien inicialmente se pensó que éste era el único compuesto intermedio, trabajos posteriores demostraron que por debajo de $1100^{\circ} \mathrm{C}$ la fase estable tiene también estructura ortorrómbica pero su composición puede describirse por la fórmula $\mathrm{ZrTi}_{2} \mathrm{O}_{6}$. Partiendo de la fase de alta temperatura, la transición entre estas dos fases tiene lugar a través de una fase ordenada, de composición $\mathrm{ZrTiO}_{4} \mathrm{ss}$, que se forma por un ordenamiento gradual de los cationes que forman la red cristalina de la fase desordenada de alta temperatura. Este ordenamiento gradual da lugar a una reducción de la longitud del eje cristalográfico b. El grado de orden depende fuertemente de la velocidad de enfriamiento utilizada para la obtención del material, lo que ha dado lugar a una aparente discrepancia en los resultados de distintos autores. De hecho, no fue hasta el año 2005 cuando Troitzsch y Ellis publicaron el diagrama de la figura 6 en el cual la transición de fases propuestas explica los resultados de los autores anteriores.

En cuanto al sistema ternario $\mathrm{ZrO}_{2}-\mathrm{TiO}_{2}-\mathrm{Y}_{2} \mathrm{O}_{3}$, únicamente se han publicado secciones isotermales a temperaturas entre 1000 y $1700^{\circ} \mathrm{C}$. La temperatura sobre la que ha trabajado un número mayor de autores es $1500^{\circ} \mathrm{C}$. Como muestra la figura 11, los resultados publicados son, en general, compatibles, con pequeñas diferencias en los límites de compatibilidad de las distintas regiones. La única discrepancia significativa se refiere a la región de compatibilidad bifásica entre $\mathrm{c}-\mathrm{ZrO}_{2} \mathrm{ss}-$ $\mathrm{ZrTiO}_{4} \mathrm{ss}$, propuesta por la mayoría de los autores, la cual es modificada por Feighery y col. en una región trifásica de coexistencia de $\mathrm{c}-\mathrm{ZrO}_{2} \mathrm{ss}-\mathrm{ZrTiO}_{4} \mathrm{ss}-\mathrm{Y}_{2} \mathrm{Ti}_{2} \mathrm{O}_{7} \mathrm{ss}$ y una región bifásica de coexistencia de $\mathrm{ZrTiO}_{4} \mathrm{ss}-\mathrm{Y}_{2} \mathrm{Ti}_{2} \mathrm{O}_{7} \mathrm{ss}$.

Todos los estudios sobre expansión térmica de materiales con la composición estequiométrica, $\mathrm{ZrTiO}_{4^{\prime}}$ describen anisotropía cristalográfica. Sin embargo, el grado de anisotropía depende de la historia térmica de los materiales caracterizados. Este hecho se explica por la transición gradual orden-desorden entre las fases de baja y alta temperatura. Así, dependiendo de la temperatura de tratamiento del material y de la velocidad de enfriamiento, las estructuras cristalinas obtenidas, con diferentes longitudes del eje b, presentarán diferentes grados de expansión térmica en este eje. De hecho, como se muestra en la tabla 1, los coeficientes de expansión térmica de los ejes a y c para diferentes materiales son similares mientras que se aprecian diferencias significativas en el eje b.

Dada la anisotropía de la expansión térmica cristalográfica de este compuesto, podría ser planteado como constituyente de componentes estructurales. En general, para asegurar la integridad estructural y la homogeneidad microestructural de una pieza cerámica, es preciso utilizar velocidades de enfriamiento desde la temperatura de fabricación relativamente bajas. A partir de los resultados expuestos en este trabajo, el uso de velocidades de enfriamiento compatibles con el procesamiento de piezas cerámicas tiene una importancia fundamental en el caso del titanato de circonio. La posibilidad real de uso de este compuesto como constituyente de materiales con aplicaciones estructurales exige, no sólo el conocimiento de las relaciones de equilibrio de fases, sino, también, el control de los parámetros de procesamiento para manipular las propiedades de los materiales obtenidos.

\section{AGRADECIMIENTOS}

Este trabajo ha sido desarrollado gracias a la financiación económica del Ministerio de Ciencia e Innovación (MICINN) a través del proyecto MAT 2009-14369-C02-01.

Emilio López-López agradece a la Comunidad de Madrid y al Fondo Social Europeo la financiación económica a través del contrato CPI / 0552/ 2007.

\section{BIBLIOGRAFÍA}

1. F. H. Brown and P. Duwez, "The zirconia-titania system," J. Am. Ceram. Soc., vol. 37, pp. 129-132, 1954.

2. L. W. Coughanour, R. S. Roth, and V. A. DeProsse, "Phase equilibrium relations in the systems lime-titania and zirconia-titania," J. Res. Nat. Bur. Stand., vol. 52, pp. 37-42, 1954.

3. U. Troitzsch and D. J. Ellis, "High-PT study of solid solutions in the system $\mathrm{ZrO}_{2}-\mathrm{TiO}_{2}$ :The stability of srilankite," Eur. J. Mineral, vol. 16, pp. 577-584, 2004 . 
4. A. E. McHale and R. S. Roth, "Low-temperature phase relationships in the system $\mathrm{ZrO}_{2}-\mathrm{TiO}_{2}$ "' J. Am. Ceram. Soc., vol. 69, pp. 827-832, 1986.

5. A. Willgallis, E. Seigmann, and T. Hettiarachi, "Srilankite, a new Zr-Ti oxide mineral," N. Jb. Miner. Mh., vol. 4, pp. 151-157, 1983.

6. R. E. Newnham, "Crystal Structure of $\mathrm{ZrTiO}_{4^{\prime}}$ ” J. Am. Ceram. Soc., vol. 50, pp. 216, 1967.

7. P. Bordet, A. McHale, A. Santoro, and R. S. Roth, "Powder neutron diffraction study of $\mathrm{ZrTiO}_{4^{\prime}} \mathrm{Zr}_{5} \mathrm{Ti}_{7} \mathrm{O}_{24}$ and $\mathrm{FeNb}_{2} \mathrm{O}_{6^{\prime}}$ " J. Solid State Chem., vol. 64 , pp. 30-46, 1986.

8. U. Troitzsch, A. G. Christy, and D. J. Ellis, "The crystal structure of disordered $(\mathrm{Zr}, \mathrm{Ti}) \mathrm{O}_{2}$ solid solution including srilankite: evolution towards tetragonal $\mathrm{ZrO}_{2}$ with increasing $\mathrm{Zr}$," Phys. Chem. Minerals, vol. 32, pp. 504-514, 2005.

9. N. Vittayakorn, "Synthesis and a crystal structural study of microwave dielectric zirconium titanate $\left(\mathrm{ZrTiO}_{4}\right)$ powders via a mixed oxide synthesis route," J. Ceram. Process. Res., vol. 7, pp. 288-291, 2006.

10. Y. Park and Y. Kim, "Influence on cooling rate on the physical properties of tin modified zirconium titanate," J. Mater. Sci. Lett., vol. 15, pp. 853-855, 1996.

11. G. Wolfram and H. E. Göbel, "Existence range, structural and dielectric properties of $\mathrm{Zr}_{\mathrm{x}} \mathrm{Ti}_{\mathrm{y}} \mathrm{SnzO}_{4}$ ceramics $(\mathrm{X}+\mathrm{Y}+\mathrm{Z}=2)$," Mater. Res. Bull., vol. 16, pp. 1455-1463, 1981.

12. A. Bianco, G. Gusmano, R. Freer, and P. Smith, "Zirconium titanate microwave dielectrics prepared via polymeric precursor route," J. Eur Ceram. Soc, vol. 19, pp. 959-963, 1999.

13. S. Hirano, T. Hayashi, and A. Hattori, "Chemical processing and mircrowave characteristic of $(\mathrm{Zr}, \mathrm{Sn}) \mathrm{TiO}_{4}$ microwave dielectrics," J. Am. Ceram. Soc., vol. 74, pp. 1320-1324, 1991.

14. R. Christoffersen, P. K. Davies, X. Wei, and T. Negas, "Effect of Sn substitution on cation ordering in $\left(\mathrm{Zr}_{1-x} \mathrm{Sn}_{\chi}\right) \mathrm{TiO}_{4}$ microwave dielectric ceramics," J. Am. Ceram. Soc., vol. 77, pp. 441-450, 1994.

15. P. Victor, S. Bhattacharyya, and S. B. Krupanidhi, "Dielectric relaxation in laser ablated polycrystalline $\mathrm{ZrTiO}_{4}$ thin films," J. Appl. Phys., vol. 94, pp. 5135-5142, 2003.

16. S. W. Liu, C. F. Song, M. K. Lü, S. F. Wang, D. L. Sung, Y. X. Qi, D. Xu, and D. R. Yuan, "A novel $\mathrm{TiO}_{2} / \mathrm{Zr}_{\mathrm{x}} \mathrm{Ti}_{1-\mathrm{x}} \mathrm{O}_{2}$ composite photocatalytic films," Catal. Commun., vol. 4, pp. 343-346, 2003.

17. B. M. Reddy and A. Khan, "Recent advances on $\mathrm{TiO}_{2}-\mathrm{ZrO}_{2}$ miexed oxides as catalysts and catalyst supports," Catalysis reviews: Sci. and Eng., vol. 47, pp. 257-296, 2005.

18. Y. S. Mazurkevich and I. M. Kobasa, " $\mathrm{ZrO}_{2}-\mathrm{TiO}_{2}$ materials," Inorg. Mater., vol. 37, pp. 1285-1288, 2001.

19. J. R. Sohn and S. H. Lee, "Effect of $\mathrm{TiO}_{2}-\mathrm{ZrO}_{2}$ composition on catalytic activity of supported $\mathrm{NiSO}_{4}$ for ethylene dimerization," Appl. Catal. A: Gen, vol. 321, pp. 27-34, 2007.

20. J. C. Wu, C.S. Chung, C. L. Ay, and I. Wang, "Nonoxidative dehydrogenation of ethylbenzene over $\mathrm{TiO}_{2}-\mathrm{ZrO}_{2}$ catalysts," J. Catal., vol. 87, pp. 98-107, 1984.

21. C. E. F. Costa, C. L. Samara, S. J. G. Lima, C. A. Paskocimas, E. Longo, V. J. F. Jr., A. S. Araújo, I. M. G. Santos, and A. G. Souza, "Synthesis and thermal characterization of zirconium titanate pigments," J. Them. Anal. Cal., vol. 75, pp. 467-473, 2004.

22. S. Rengakuji, Y. Nakamura, and Y. Hara, "Preparation hydrocarbon sensing properties of Ti-Zr-O thin film," Electrochemistry, vol. 69, pp. 764-768, 2001.

23. A. Gajovic, A. Santic, I. Djerdj, N. Tomasic, A. Mogus-Milankovic, and D. $\mathrm{S}$. Su, "Structure and electrical conductivity of porous zirconium titanate ceramics produced by mechanochemical treatment and sintering," J. Alloy Compd., vol. 479, pp. 525-531, 2009.

24. B. K. Hom, R. Stevens, B. F. Woodfield, J. Boerio-Goates, R. L. Putnam, K. B. Helean, and A. Navrotsky, "The thermodynamics of formation, molar heat capacity, and thermodynamic functions of $\mathrm{ZrTiO}_{4}(\mathrm{cr}), " \mathrm{~J}$. Chem. Thermodynamics, vol. 33, pp. 165-178, 2001.

25. I. J. Kim and H. C. Kim, "Zero level thermal expansion materials based on $\mathrm{ZrTiO}_{4}-\mathrm{Al}_{2} \mathrm{TiO}_{5}$ ceramics synthesized by reaction sintering," J. Ceram. Process. Res., vol. 5, pp. 308-312, 2004.

26. S. Ananta, R. Tipakontitikul, and T. Tunkasiri, "Synthesis, formation and characterization of zirconium titanate (ZT) powders," Mater. Lett., vol. 57, pp. 2637-2642, 2003.

27. V. Licina, A. Gajovic, A. Mogus-Milankovic, I. Djerdj, N. Tomastic, and D. $\mathrm{Su}$, "Correlation between the microstructure and the electrical properties of $\mathrm{ZrTiO}_{4}$ ceramics," J. Am. Ceram. Soc., vol. 91, pp. 178-186, 2008.

28. A. K. Bhattacharya, K. K. Mallick, A. Hartridge, and J. L. Woodhead, "Sol gel preparation, structure and thermal stability of crystalline zirconium titanate microspheres," J. Mater. Sci. Lett., vol. 31, pp. 267-271, 1996.

29. E. López-López, C. Baudín, and R. Moreno, "Synthesis of zirconium titanate-based materials by colloidal filtration and reaction sintering," Int J. Appl. Ceram. Technol., vol. 5, pp. 394-400, 2008.
30. E. López-López, C. Baudín, and R. Moreno, "Thermal expansion of zirconia-zirconium titanate materials obtained by slip casting of mixtures of Y-TZP-TiO ${ }_{2}^{\prime \prime}$ J. Eur. Ceram. Soc, vol. 29, pp. 3219-3225, 2009.

31. E. López-López, M. L. Sanjuán, R. Moreno, and C. Baudín, "Phase evolution in reaction sintered zirconium titanate based materials," J. Eur. Ceram. Soc, vol. 30, pp. 981-991, 2010.

32. H. G. Sowman and A. I. Andrews, "A study of the phase relations of $\mathrm{ZrO}_{2}$ $\mathrm{TiO}_{2}$ and $\mathrm{ZrO}_{2}-\mathrm{TiO}_{2}-\mathrm{SiO}_{2}$ " J. Am. Ceram. Soc., vol. 34, pp. 298-301, 1951.

33. T. Noguchi and M. Mizuno, "Phase changes in the $\mathrm{ZrO}_{2}-\mathrm{TiO}_{2}$ system," B. Chem. Soc. Jpn., vol. 41, pp. 2895-2899, 1968.

34. A. Cocco and G. Torriano, "Relations between the solid phases in the system $\mathrm{ZrO}_{2}-\mathrm{TiO}_{2}$ " Ann. Chim. (Rome), vol. 55, pp. 153-163, 1965

35. A. V. Shevchenko, L. M. Lopato, I. M. Maister, and O. S. Gorbunov, "The $\mathrm{TiO}_{2}-\mathrm{ZrO}$ System," Russ. J. Inorg. Chem., vol. 25, pp. 1379-1381, 1980.

36. M. J. Bannister and J. M. Barnes, "Solubility of $\mathrm{TiO}_{2}$ in $\mathrm{ZrO}_{2}$," J. Am. Ceram. Soc., vol. 69, pp. C269-C271, 1986.

37. A. E. McHale and R. S. Roth, "Investigation of the phase transition in $\mathrm{ZrTiO}_{4}$ and $\mathrm{ZrTiO}_{4}-\mathrm{SnO}_{2}$ solid solutions," J. Am. Ceram. Soc., vol. 66, pp. C18-C20, 1983.

38. A. Willgallis and $\mathrm{H}$. Hartl, " $\left(\mathrm{Zr}_{0.33} \mathrm{Ti}_{0.67}\right) \mathrm{O}_{2}-\mathrm{A}$ natural zirconium-titanium oxide with an alpha- $\mathrm{PbO}_{2}$ structure," Z. Kristallogr., vol. 164, pp. 59-66, 1983.

39. U. Troitzsch and D. J. Ellis, "The $\mathrm{ZrO}_{2}-\mathrm{TiO}_{2}$ phase diagram," J. Mater. Sci., vol. 40, pp. 4571-4577, 2005.

40. K. Tsukuma, "Transparent titania-yttria-zirconia ceramics," J. Mater. Sci. Lett., vol. 5, pp. 1143-1144, 1986.

41. C. L. Lin, D. Gan, and P. Shen, "The effects of $\mathrm{TiO}_{2}$ addition on the microstructure and transformation of $\mathrm{ZrO}_{2}$ with 3 and 6 mol. $\% \mathrm{Y}_{2} \mathrm{O}_{3}{ }^{\prime \prime}$ Mater. Sci. Eng. A, vol. 129, pp. 147-155, 1990.

42. W. Pyda, K. Haberko, and M. M. Bucko, "A study on preparation of tetragonal zirconia polycrystal (TZP) in the $\mathrm{TiO}_{2}-\mathrm{Y}_{2} \mathrm{O}_{3}-\mathrm{ZrO}_{2}$ system," Ceram. Int., vol. 18, pp. 321-326, 1992.

43. H. Yokokawa, N. Sakai, T. Kawada, and M. Dokiya, "Phase diagram calculations for $\mathrm{ZrO}_{2}$ based ceramics:thermodynamic regularities in zirconate foramtion and solubilities of transition metal oxides," Sci. and technol. zirconia V. Ed. by S.P.S. Badwal, M. J. Bannister and R. H. J. Hannink, Technomic Publishing, Lancaster, Pennsylvania, pp. 59-68, 1993.

44. S. S. Liou and W. L. Worrel, "Electrical properties of novel mixed conducting oxides," Appl. Phys. A., vol. 49, pp. 25-31, 1989.

45. H. Yokokawa, N. Sakai, T. Kawada, and M. Dokiya, "Phase diagram calculations for $\mathrm{ZrO}_{2}$ based ceramics:thermodynamic regularities in zirconate foramtion and solubilities of transition metal oxides," Sci. and technol. zirconia V. Ed. by S.P.S. Badwal, M. J. Bannister and R. H. J. Hannink, Technomic Publishing, Lancaster, Pennsylvania, pp. 59-68, 1993.

46. M. T. Colomer, P. Durán, A. Caballero, and J. R. Jurado, “Microstructure, electrical porpoerties and phase equilibria relationships in the $\mathrm{ZrO}_{2}-\mathrm{Y}_{2} \mathrm{O}_{3}$ $\mathrm{TiO}_{2}$ system: the subsolidus isothermal section at $1500^{\circ} \mathrm{C}^{\prime \prime}$ Mater. Sci. Eng. A, vol. 229, pp. 114-122, 1997.

47. L. S. M. Traqueia, T. Pagnier, and F. M. B. Marques, "Structural and electrical characterization of titania-doped YSZ," J. Eur. Ceram. Soc, vol. 17, pp. 1019-1026, 1997.

48. K. Kobayashi, K. Kato, K.Terabe, S.Yamaguchi, and Y.Iguchi, "Phase relation of $\mathrm{ZrO}_{2}-\mathrm{YO}_{15}-\mathrm{TiO}_{2}$ ceramics prepared by sol-gel method," J. Ceram. Soc. Jpn., vol. 106, pp. 860-866, 1998.

49. A. J. Feighery, J. T. S. Irvine, D. P. Fagg, and A. Kaiser, "Phase relations at $1500^{\circ} \mathrm{C}$ in the ternary system $\mathrm{ZrO}_{2}-\mathrm{Y}_{2} \mathrm{O}_{3}-\mathrm{TiO}_{2}$," J. Solid State Chem., vol. 143, pp. 273-276, 1999.

50. T. A. Schaedler, O. Fabrichnaya, and C. G. Levi, "Phase equilibria in the $\mathrm{TiO}_{2}-\mathrm{YO}_{15}-\mathrm{ZrO}_{2}$ system," J. Eur. Ceram. Soc, vol. 28, pp. 2509-2520, 2008.

51. D. P. Fagg, J. R. Frade, M. Mogensen, and J. T. S. Irvine, "Effects of firing schedule on solubility limits and transport properties of $\mathrm{ZrO} 2-\mathrm{TiO} 2-\mathrm{Y} 2 \mathrm{O} 3$ fluorites," J. Solid State Chem., vol. 180, pp. 2371-2376, 2007.

52. C. Baudín, "Resistencia de los refractarios al choque térmico. I: Aproximación termoelástica y criterio de balance energético," Bol. Soc. Esp. Ceram. V., vol. 32, pp. 237-244 (1993).

53. C. Baudín, "Resistencia de los refractarios al choque térmico. II: Teoría unificada de Hasselman," Bol. Soc. Esp. Ceram. V., vol. 32, pp. 293-298 (1993).54. R. W. Lynch and B. Morosin, "Thermal expansion, compressibility, and polymorphism in hafnium and zirconium titanates," J. Am. Ceram. Soc., vol. 55, pp. 409-413, 1972

55. H. Ikawa, A. Iwai, K. Hiruta, H. Shimojima, K. Urabe, and S. Udagawa, "Phase transformation and thermal expansion of zirconium and hafnium titanates and their solid solutions," J. Am. Ceram. Soc., vol. 71, pp. 120-127, 1988.

56. Y. Park, "Thermal expansion and cooling rate dependence of transition temperature in ZrTiO4 single crystal," Mater. Res. Bull, vol. 33, pp. 13251329, 1998.

Recibido: $24 / 03 / 2011$

Aceptado: 22/07/2011 